\title{
Entre mémoire carolingienne et réforme « grégorienne »
}

Stratégies discursives, identité monastique et enjeux de pouvoir à Redon aux XIe et XIIe siècles

Between Carolingian legacy and Gregorian reform. Discursive strategies, monastic identity and power struggles in Redon in the eleventh and twelfth centuries

\section{Florian Mazel}

\section{(2) OpenEdition}

\section{Journals}

Édition électronique

URL : http://journals.openedition.org/abpo/2954

DOI : $10.4000 / a b p o .2954$

ISBN : 978-2-7535-4130-6

ISSN : 2108-6443

Éditeur

Presses universitaires de Rennes

Édition imprimée

Date de publication : 15 avril 2015

Pagination : 9-39

ISBN : 978-2-7535-4128-3

ISSN : 0399-0826

\section{Référence électronique}

Florian Mazel, « Entre mémoire carolingienne et réforme « grégorienne » », Annales de Bretagne et des Pays de l'Ouest [En ligne], 122-1 | 2015, mis en ligne le 15 avril 2017, consulté le 19 avril 2019. URL: http://journals.openedition.org/abpo/2954; DOI : 10.4000/abpo.2954 


\title{
Entre mémoire carolingienne et réforme "grégorienne"
}

\author{
Stratégies discursives, identité monastique \\ et enjeux de pouvoir à Redon aux XI ${ }^{\mathrm{e}}$-XII ${ }^{\mathrm{e}}$ siècles
}

\author{
Florian MAZEL \\ Université Rennes 2 - CERHIO UMR 6258, Institut universitaire de France
}

Après un premier essor dans les années 832-850, les déchirements du regnum breton, les troubles de l'Empire et les raids normands plongèrent l'abbaye Saint-Sauveur de Redon dans une période difficile. Dès le début des années 860, une partie des moines avaient gagné la plebs Lan (aujourd'hui Plélan-le-Grand), où le roi Salomon avait fondé un monastère dans l'un des ses palais et où les moines se trouvaient plus à l'abri des agressions scandinaves que dans la basse vallée de la Vilaine ${ }^{1}$. C'est d'ailleurs là qu'après avoir renoncé à sa charge l'abbé fondateur Conwoion mourut le 5 janvier 868 et fut inhumé ${ }^{2}$. La communauté se rassembla toutefois sans doute de nouveau à Redon après la mort de Salomon († 874) : on connaît le nom de plusieurs de ses abbés et l'on a conservé un certain nombre de dona-

1. Sur la politique de Salomon envers Redon, voir en dernier lieu BRETT Caroline, "Redon, une abbaye carolingienne ", dans Pichot, Daniel, Provost, Georges (dir.), Redon, de l'abbaye à la ville, à paraître à Rennes fin 2015 (avec renvoi à la bibliographie antérieure). Le statut exact de Plélan, des années 860 à la mort de Salomon en 874, reste difficile à cerner. Pour les moines de Redon, ce nouveau monastère fut sans doute toujours conçu comme une dépendance et un refuge. En revanche, pour Salomon, premier et seul rex des Bretons, au regard de l'importance du site (un ancien palais), de la dédicace au Sauveur (comme Redon), de la donation d'éminentes reliques (celles de saint Maixent, "luctus Aquitaniae, lux, laus honorque Britanniae "), enfin du choix d'y établir sa sépulture aux côtés de son épouse Guenwreth et de l'abbé Conwoion (Cartulaire de l'abbaye de Redon, éd. A. de Courson, Paris, 1863 [désormais CR], n² 241; trad. fr. dans Documents de l'histoire de la Bretagne, éd. J. Delumeau, Toulouse, 1971, p. 84), il s'agissait vraisemblablement d'une nouvelle fondation, concurrente de Redon qu'il estimait sans doute trop liée à ses prédécesseurs Nominoé et Érispoé et aux empereurs carolingiens Louis le Pieux et Charles le Chauve. Sur la fondation de Plélan, voir également GuIGon Philippe, Les églises du haut Moyen Âge en Bretagne, Saint-Malo, 1997-1998, t. 2, p. 142-145.

2. $C R, \mathrm{n}^{\circ} 241$ et Vita sancti Conuuoinuis, éd. Caroline BreTt, The Monks of Redon : Gesta sanctorum Rotonensium and Vita Conwoionis, Woodbridge, 1989, p. 245. 
tions concernant explicitement Redon entre les années 870 et $910 / 913^{3}$. Puis vient le temps du silence documentaire : à l'exception d'une notice de 924 recopiée dans le cartulaire mais sans rapport direct avec Redon, plus aucun acte n'est conservé avant la fin du $\mathrm{X}^{\mathrm{e}}$ siècle $^{4}$. Cette lacune a généralement été interprétée comme le signe d'un abandon du site, voire d'une disparition de la communauté ${ }^{5}$. L'un comme l'autre sont peu probables. La région, en effet, ne fut jamais totalement abandonnée par ses élites, même entre 913 et 936 , au temps des plus rudes attaques normandes. Le comte de Rennes Bérenger notamment est resté sur place et la ville de Rennes ne figure jamais au nombre des cités évacuées par leur clergé telles Nantes, Vannes, Alet ou Dol. Flodoard mentionne l'alliance que le comte de Rennes aurait conclu avec Guillaume Longue-Épée, comte de Rouen, en 931, alors qu'Alain II, petit-fils d'Alain le Grand, avait pour sa part trouvé refuge en Angleterre. Après qu'en 936-937 Alain II a repris pied à Nantes, Bérenger combat les Normands à ses côtés à Trans en 939. Les décennies suivantes sont plus obscures et la rivalité entre les comtes de Rennes, de Nantes et d'Angers, qui domine les années 960-990, n'a pu que nuire à l'abbaye située aux confins des évêchés de Vannes, Rennes et Nantes ${ }^{6}$.

Quoi qu'il en soit, ce n'est qu'avec l'établissement de l'hégémonie de la maison de Rennes, à la fin du $\mathrm{x}^{\mathrm{e}}$ siècle, que Redon réapparaît dans la documentation. Elle est alors clairement située dans l'orbite des comtes rennais qui se sont appropriés le titre ducal : Thibaud, le premier abbé qui nous soit connu depuis le début du siècle et qui est mentionné dans un acte des années 990-992, appartient vraisemblablement à une famille rennaise

3. Il s'agit des abbés Libère, Fulcric, Bernard, Hailcobrant, Catluiant (cf. GuILLOTEL Hubert, "Le manuscrit ", dans Cartulaire de l'abbaye Saint-Sauveur de Redon, éd. fac-similé, Rennes, 1998, p. 9-25, ici p. 23). En 878, Alain le Grand est lui-même présent à Redon où il fait donation de la plebs de Bouvron ( $\left.C R \mathrm{n}^{\circ} 238\right)$. Pour la redatation des derniers actes " carolingiens " de Redon, voir GuILLOTEL, Hubert, " Répertoire chronologique ", ibidem, p. 71-78, ici p. 76. Passé 874, Plélan n'est qu'une cella dépendant de Redon (cf. BRETT, Caroline, The Monks of Redon, op. cit., p. 13-14, qui rapporte l'opinion semblable de Wendy Davies).

4. $C R \mathrm{n}^{\circ} 283$. Cette notice raconte le retour des reliques de saint Maixent de Plélan à Poitiers.

5. Voir par exemple DEUFFIC, Jean-Luc, « L'exode des corps saints hors de Bretagne : des reliques au culte liturgique ", Pecia, 8-11, 2005, p. 355-424, ici p. 357-358. Cette tenace tradition historiographique a même avancé, sur la base d'une lecture erronée de la notice de 924, malheureusement confortée par Hubert Guillotel ("L'exode du clergé breton devant les invasions scandinaves ", Mémoires de la Société d'histoire et d'archéologie de Bretagne, 59, 1982, p. 269-315), que tous les moines de Redon avaient accompagné les reliques de saint Maixent en Poitou et qu'ils s'étaient soumis temporairement à l'autorité de l'abbé de Saint-Maixent Adhémar, avant de revenir en Bretagne dans des conditions obscures dans la seconde moitié du $\mathrm{x}^{\mathrm{e}}$ siècle. Sur cette notice, voir l'excursus à la fin de cet article.

6. Sur ces événements, voir GuILLOTEL, Hubert, La Bretagne des saints et des rois, ve$X^{e}$ siècle, Rennes, 1984, p. 385-389 et 393-395; Id., " Le premier siècle du pouvoir ducal breton (936-1040)", dans les actes du 103e Congrès national des sociétés savantes (Nancy-Metz, 1978), Paris, 1979, p. 63-84; Smith, Julia, Province and Empire. Brittany and the Carolingians, Cambridge, 1992, p. 187-206. 
de l'entourage comtal ${ }^{7}$; Mainard, abbé d'avant 1008 à avant 1019, qui fut également abbé du Mont-Saint-Michel jusqu'en 1009, était proche du duc Geoffroy I ${ }^{\mathrm{er}}(992-\uparrow 1008)$ qui l'avait probablement sollicité pour réformer Redon ${ }^{8}$; Catwallon enfin, abbé des environs de 1019 à 1041, était un frère de Geoffroy I ${ }^{\mathrm{er}}$ et donc l'oncle d'Alain III (1008- $\left.\uparrow 1040\right)^{9}$. Dans ce contexte, Redon semble connaître une sorte de second départ, dont l'essor patrimonial et la reconstruction de l'abbatiale constituent les meilleurs indices ${ }^{10}$. Ce renouveau reste toutefois délicat à apprécier en raison de la rareté des actes antérieurs à 1050 et du grand nombre d'actes faux ou interpolés. Quelles sont en effet les sources à notre disposition? Si on laisse de côté les quelques chartes émanant des évêques de Vannes et Nantes ou d'abbayes voisines (le Mont-Saint-Michel, Marmoutier et Quimperlé), il s'agit de trois documents produits à Redon même :

1 - Un texte hagiographique d'abord, la Vita Conwoionis, consacrée à l'abbé fondateur, composée au $\mathrm{XI}^{\mathrm{e}}$ siècle et plus probablement dans la seconde moitié du siècle, à des fins sans doute d'abord liturgiques ${ }^{11}$;

2 - Le cartulaire de Redon ensuite, dont la première phase rédactionnelle, due à l'initiative de l'abbé Aumod (1062-†1083), commence au tournant des années 1070 et des années $1080^{12}$. Cette première phase nous est

7. $C R \mathrm{n}^{\circ} 329$ et $357=$ Guillotel, Hubert, Actes des ducs de Bretagne, 944-1148, Rennes, $2014, n^{\circ} 7$ (datation corrigée). Voir également Gullotel, Hubert, "Le manuscrit ", p. 23-24.

8. Voir la notice de GAZEAU, Véronique, Normannia monastica. Prosopographie des abbés bénédictins ( $X^{e}$-XII siècle), Caen, 2007, p. 198-200, avec renvoi à la bibliographie antérieure.

9. $C R \mathrm{n}^{\circ} 323$ = Gullotel, Hubert, Actes des ducs de Bretagne, op. cit., $\mathrm{n}^{\circ} 11$; Chronique de Quimperlé, éd. L. Maître et P. de Berthou, Cartulaire de l'abbaye Sainte-Croix de Quimperlé, Paris, 1904, p. 103.

10. Sur l'essor seigneurial de l'abbaye, voir les aperçus de CHÉDEvILLE, André, "Société et économie ", dans Cartulaire de Saint-Sauveur, op. cit., p. 38-47. Sur la reconstruction de l'abbatiale, voir DÉcEneux, Marc, La Bretagne romane, Rennes, 1998, p. 36-39, et Id., "Quelques édifices du premier art roman breton : éléments pour une nouvelle chronologie ", Dossiers du CeRAA, 32, 2004, p. 73-102.

11. Bibliotheca hagiographica latina, éd. société des Bollandistes, Bruxelles, 1898-1899, n ${ }^{\circ}$ 1946, éd. BRETT, Caroline, The Monks of Redon, op. cit., p. 226-245. Ce texte est considéré par Caroline Brett comme de peu antérieur au milieu du XI ${ }^{\mathrm{e}}$ siècle en raison de liens étroits avec la Chronique de Nantes et les Annales dites de Redon (en fait probablement du Mont-Saint-Michel) : BRETT, Caroline, The Monks of Redon, op. cit., p. 18. Je suis personnellement plus sensible aux analyses de Joseph-Claude Poulin qui, sur la base de la tonalité grégorienne de certains passages (que Caroline Brett a elle-même remarquée : ibidem, p. 18), le date plutôt de la seconde moitié du XI ${ }^{\mathrm{e}}$ siècle : Poulin, Joseph-Claude, « Le dossier hagiographique de saint Conwoion de Redon. À propos d'une édition récente ", Francia, 18/1, 1991, p. 139-159, ici p. 158; Id., L'hagiographie bretonne du haut Moyen Âge. Répertoire raisonné, Ostfildern, 2009, p. 85-97, ici p. 97. Il est en tout cas antérieur à la fin $\mathrm{du} \mathrm{XI}^{\mathrm{e}}$ siècle puisque c'est à cette époque que furent perdus le commencement et la fin des Gestes des saints de Redon encore connus de l'auteur de la Vita.

12. Guillotel Hubert, "Le manuscrit ", art. cit., p. 15-16. Il s'agit des fol. 1 à 139 (=CR, $\mathrm{n}^{\circ} 1$ à 289). Les dernières transcriptions du moine Judicaël concernent des actes de 1081 et l'abbé Aumod décède un 5 septembre entre 1081 et 1084. L'acte suivant, copié par le moine Guigon, date du 30 décembre 1089. Sur ce cartulaire, voir aussi GuILLOTEL Hubert, "Les cartulaires de l'abbaye de Redon ", Mémoires de la Société d'histoire et d'archéologie de Bretagne, 63, 1986, p. 27-48; DAvies Wendy, "The composition of the Redon cartulary ", 
conservée sous une forme lacunaire : il y manque les folios 9-50 correspondant à des actes des années 840. Après avoir été délaissé quelques années, le cartulaire est repris sous l'abbatiat de Robert (1085-1096), puis régulièrement enrichi jusqu'à l'abbatiat d'Yves (1144-1164) ${ }^{13}$. Ces continuations comprennent un assez grand nombre d'actes faux ou interpolés - au moins 19 - se rapportant aux $\mathrm{XI}^{\mathrm{e}}$-XII ${ }^{\mathrm{e}}$ siècles et dont l'étude systématique reste à faire (figure 1$)^{14}$.

\section{Figure 1 - Actes sincères, faux ou interpolés dans les continuations du cartulaire de Redon (NB : ne sont pas pris en compte les actes non datés par un abbatiat, soit 1 acte faux ou interpolé et 14 actes sincères)}

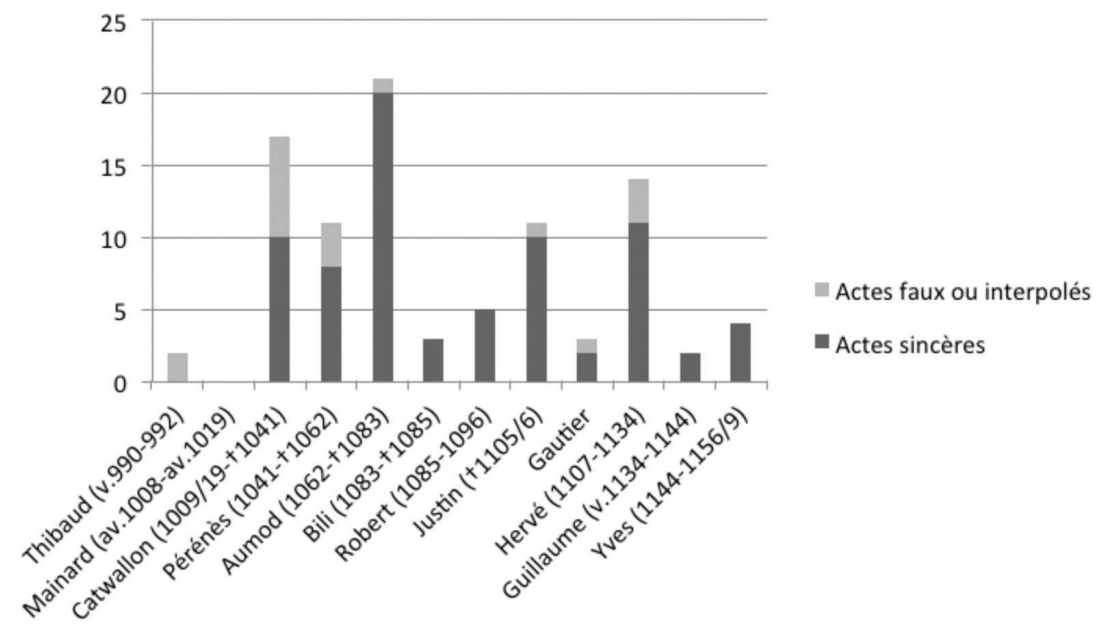

3 - Le " petit cartulaire " enfin, aujourd'hui perdu mais en partie connu grâce à quelques épaves et dont la compilation date du début du XII ${ }^{\mathrm{e}}$ siècle (après 1112 et peut-être même après 1127) ${ }^{15}$.

Si l'on accepte la datation tardive de la Vita Conwoionis, ces trois documents sont donc contemporains de la réforme " grégorienne " dont la Bretagne et plus particulièrement l'évêché de Nantes furent un terrain pré-

Francia, 17, 1990, p. 69-90; BRUNTERC'H Jean-Pierre, " La partie perdue du cartulaire de Redon ", dans Provost, Georges, LETERTRE, Raymond (dir.), Cartulaire de l'abbaye SaintSauveur de Redon, t. 2, Rennes, 2004, p. 7-31.

13. GulLLOTEL, Hubert, "Le manuscrit ", art. cit., p. 16.

14. GuILlOTEL, Hubert, "Le manuscrit », art. cit., p. 18-20 et 77-78. Il s'agit des $C R, \mathrm{n}^{\circ} 292$, 293, 294, 296, 300, 302, 305, 321, 329, 341, 347, 348, 356, 357, 370, 373, 376, 378, 385. Sur ces faux, voir aussi DAVIES Wendy, "Forgery in the Cartulaire de Redon ", dans Fälschungen im Mittelalter, t. 4, Munich, 1988, p. 265-274.

15. Voir Guillotel, Hubert, "Les cartulaires de Redon ", art. cit., et Cartulaire de l'abbaye Saint-Sauveur de Redon, t. 2, op. cit. 
coce $^{16}$. Un même contexte de rédaction lié aux initiatives de l'abbé Aumod (1062- $\uparrow 1083)$ pourrait même unir la Vita Conwoionis et la mise en route du cartulaire. Au même titre que les motifs ayant présidé à la confection des faux ou des actes interpolés, lesquels peuvent être contemporains ou antérieurs à leur transcription dans le cartulaire, ces documents permettent d'accéder, à travers les stratégies discursives mises en œuvre, à la manière dont la communauté de Redon conçoit alors son passé et entend se situer dans son environnement politique, social et institutionnel. Comme nous le verrons, ces stratégies reposent sur une mise en valeur orientée de l'héritage carolingien, associée à une certaine insistance sur l'engagement de Redon dans la réforme de l'Église promue par la papauté et un corps épiscopal rénové. Ces choix conduisent les moines de l'âge grégorien à minimiser le rôle des ducs, qui furent pourtant à l'origine du renouveau monastique au début du $\mathrm{XI}^{\mathrm{e}}$ siècle et firent de l'abbaye un haut lieu de leur pouvoir tout au long des $\mathrm{XI}^{\mathrm{e}}$-XII ${ }^{\mathrm{e}}$ siècles ${ }^{17}$.

\section{Une mise en valeur orientée de l'héritage carolingien}

Le premier objectif d'un cartulaire monastique est de rassembler en un livre unique les actes attestant des droits et biens de l'établissement dans une double finalité archivistique et juridique, ce qui en fait au premier chef un instrument de défense patrimoniale. Au regard de ce motif classique, l'originalité du cartulaire de Redon réside dans la masse des actes carolingiens qu'il contient, un cas unique dans l'espace français : on y compte en effet quelques 280 actes du IX ${ }^{\mathrm{e}}$ siècle, sans compter les actes perdus qui figuraient entre les folios 9 et $50^{18}$, contre moins d'une centaine d'actes pour les années 1000-1150 (figure 2). La phase de rédaction initiale, sous l'abbatiat d'Aumod, concerne même quasi exclusivement la documentation carolingienne. Cette masse distingue le cartulaire de Redon des opérations

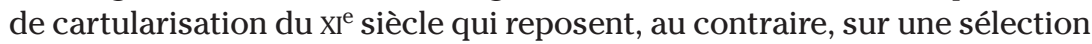
drastique de la documentation du haut Moyen Âge, en raison notamment de l'obsolescence des cadres et du lexique institutionnels et juridiques carolingiens $^{19}$. Comme l'a souligné Patrick Geary, la fabrique des cartu-

16. GuIllotel, Hubert, "Bretagne et papauté au XI ${ }^{\mathrm{e}}$ siècle ", dans L'Église de France et la papauté ( $X^{e}$-XIII siècle), Bonn, 1993, p. 265-286 et ID., " La pratique du cens épiscopal dans l'évêché de Nantes. Un aspect de la réforme ecclésiastique en Bretagne dans la seconde moitié du XI $\mathrm{e}^{\mathrm{e}}$ siècle ", Le Moyen Âge, 80, 1974, p. 5-49.

17. S'agissant du cartulaire, Hubert Guillotel avait déjà eu l'intuition d'une telle présentation orientée de l'histoire de l'abbaye et de son engagement dans ce qu'il appelait la pré-réforme grégorienne ("Le manuscrit ", art. cit., p. 17).

18. On peut estimer à une quarantaine les actes manquants, ce qui ferait un total de quelques 320 actes carolingiens pour une centaine d'actes des XIe-début XII ${ }^{\mathrm{e}}$ siècles.

19. Sur ces opérations de tri, voir notamment Chastang, Pierre, Lire, écrire, transcrire. Le travail des rédacteurs de cartulaires en Bas-Languedoc (X⿸厃-XII $e^{e}$ siècle), Paris, 2001; ID., "Des archives au codex : les enjeux de la rédaction des cartulaires (XI"-XIV e siècle) ", dans Les regroupements textuels au Moyen Âge, Paris, LAMOP, 2008 : [http://lamop.univ-paris 1.fr/ $I M G / p d f /$ article_Pierre_Chastang-2.pdf]. D'autres facteurs peuvent expliquer le travail de 
laires manifeste en général autant une volonté d'oublier le passé que d'en conserver le souvenir ${ }^{20}$.

\section{Figure 2 - Distribution chronologique des actes du cartulaire de Redon (d'après les datations proposées par H. Guillotel)}

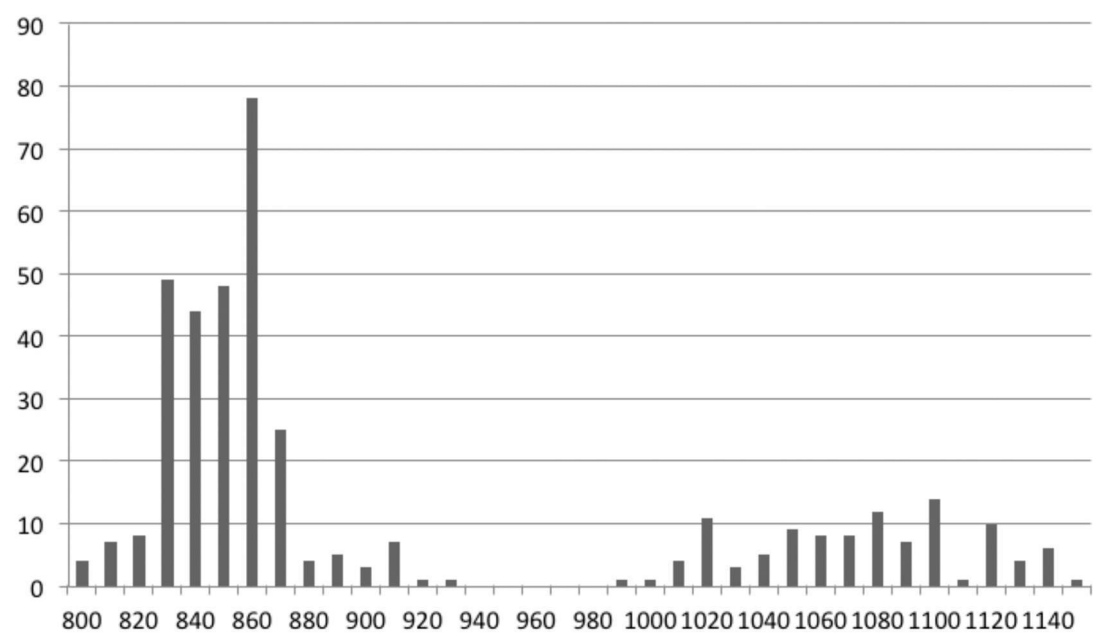

On peut d'emblée tirer deux enseignements de cette singularité, l'un matériel, l'autre idéel. Le premier conduit à relativiser la crise que la communauté monastique aurait connu au cours du $\mathrm{x}^{\mathrm{e}}$ siècle. En effet, les moines de Redon ont bien été en mesure de préserver des outrages du temps leurs archives carolingiennes, même si leur classement a sans doute subi quelques aléas ${ }^{21}$. La comparaison des actes du IX ${ }^{\mathrm{e}}$ siècle avec ceux des $\mathrm{XI}^{\mathrm{e}}$ $\mathrm{XII}^{\mathrm{e}}$ siècles d'une part, avec les bulles de confirmation pontificales d'autre part, montre par ailleurs qu'ils ont été capables de conserver l'essentiel des domaines acquis entre 832 et les années 880, en particulier ceux qui se concentraient aux alentours de l'abbaye. Ces deux constats plaident en faveur d'une présence continue des moines sur le site même de Redon tout au long du $\mathrm{x}^{\mathrm{e}}$ siècle, en dépit de la lacune documentaire, des menaces normandes et quand bien même l'abbaye n'a semble-t-il plus joué à cette époque qu'un rôle secondaire.

sélection des cartularistes : les ruptures toponymiques qui troublent l'identification des domaines et des lieux, le renouvellement des élites et l'effacement des enjeux mémoriaux propres à certaines donations...

20. GEARY, Patrick, La mémoire et l'oubli à la fin du premier millénaire, Paris, 1996.

21. Les moines ont à l'évidence conservé des originaux, mais également déjà des copies plus ou moins fidèles : cf. Gulllotel, Hubert, "Le manuscrit ", art. cit., p. 17. Sur les problèmes de classements : ibid. 
Le second enseignement tient à la place exceptionnelle accordée au passé carolingien dans la fabrique de l'identité monastique dans la deuxième moitié du $\mathrm{XI}^{\mathrm{e}}$ siècle. Ceci concerne aussi bien le récit des origines - le cartulaire présente en ouverture l'essentiel des pièces du dossier de fondation $^{22}$, puis suit d'abord un ordre globalement chronologique ${ }^{23}-$, ce qui n'est guère original, que la constitution du patrimoine, ce qui l'est beaucoup plus. Cette mise en valeur de l'époque carolingienne apparaît comme un choix de l'abbé Aumod qui semble avoir volontairement négligé les actes attestant du renouveau abbatial depuis le début du XI ${ }^{\mathrm{e}}$ siècle, dont certains seront recopiés à l'initiative de ses successeurs à la suite des actes carolingiens du premier cartulaire ou dans le " petit cartulaire ». D'ailleurs, comme le suggère une rapide comparaison de l'ensemble des actes du premier cartulaire avec l'enumeratio bonorum de la bulle d'Eugène III de 1147 , le cartulaire ne reflète pas l'ensemble de la seigneurie de Redon, ni au début des années 1080, date de la première phase rédactionnelle, ni au milieu du XII ${ }^{\mathrm{e}}$ siècle, période des derniers ajouts sous l'abbatiat d'Yves (1144-1156/1159) ${ }^{24}$. Cette enumeratio donne en effet à voir un patrimoine plus vaste et bien plus disséminé, faisant de Redon la seule abbaye bénédictine de la région à disposer d'un temporel assez bien réparti entre haute et basse Bretagne, en dépit de la prépondérance des évêchés de Nantes et Vannes (figure 3$)^{25}$. En tout cas, au milieu du XII ${ }^{\mathrm{e}}$ siècle, la seigneurie de Redon ne se limite plus à l'aire privilégiée par l'implantation carolingienne que valorisait la rédaction initiale du cartulaire. En outre, cette seigneurie apparaît structurée de manière différente, non plus autour des plebes comme au $\mathrm{IX}^{\mathrm{e}}$ siècle, mais d'un réseau de prieurés (cellae ou obedientiae) qui se constitue peu à peu depuis le début du XI ${ }^{\mathrm{e}}$ siècle. Le cartulaire surévalue donc l'héritage carolingien alors même que celui-ci ne correspond plus vraiment, et de moins en moins, à l'horizon spatial ou à l'organisation institutionnelle de la seigneurie monastique. Mais pourquoi procède-t-il ainsi? Il faut en la matière se résoudre au jeu des hypothèses.

Une première raison figure certainement dans la volonté de l'abbé Aumod de réassurer l'emprise de l'abbaye sur ses domaines les plus anciens, notamment parce qu'ils se déployaient à proximité de l'abbaye et qu'à ce titre ils étaient les plus à même de constituer une seigneurie homo-

22. Le premier quaternion $\left(f^{\circ} 1-8\right)$ s'ouvre ainsi avec la concession de l'emplacement de l'abbaye par le machtiern Ratvili $\left(C R, \mathrm{n}^{\circ} 1\right)$, le privilège de Nominoé $\left(C R, \mathrm{n}^{\circ} 2\right)$, les donations de Ratvili ( $C R, \mathrm{n}^{\circ} 3$ et 4$)$.

23. Jusqu'au $\mathrm{f}^{\circ} 76\left(C R, \mathrm{n}^{\circ} 110\right)$. Ensuite, le cartulaire suit un ordre mixte associant topographie et chronologie.

24. Cette bulle est connue par deux copies du XII ${ }^{\mathrm{e}}$ siècle figurant dans deux manuscrits du Mont-Saint-Michel qui ont sans doute transité par Redon, abbaye avec laquelle le Mont entretenait des liens étroits depuis la fin du $\mathrm{X}^{\mathrm{e}}$ siècle : le ms 82 de la bibliothèque d'Avranches (Homélies de saint Augustin), $\mathrm{f}^{\circ} 1$; le ms I de la bibliothèque de Bordeaux (une Bible), $\mathrm{f}^{\circ} 249 \mathrm{v}^{\circ}$. Elle est éditée dans les Papsturkunden in Frankreich. Neue Folge. 5. Touraine, Anjou, Maine und Bretagne, éd. J. Ramackers, Göttingen, 1956, n² 253, p. 357-359.

25. Voir les remarques d'A. Chédeville dans ChÉDEvILLE, André, TonNERRE, Noël-Yves, La Bretagne féodale, $X I^{e}$-XIII siècle, Rennes, 1987, p. 229. 
Florian MAZEL
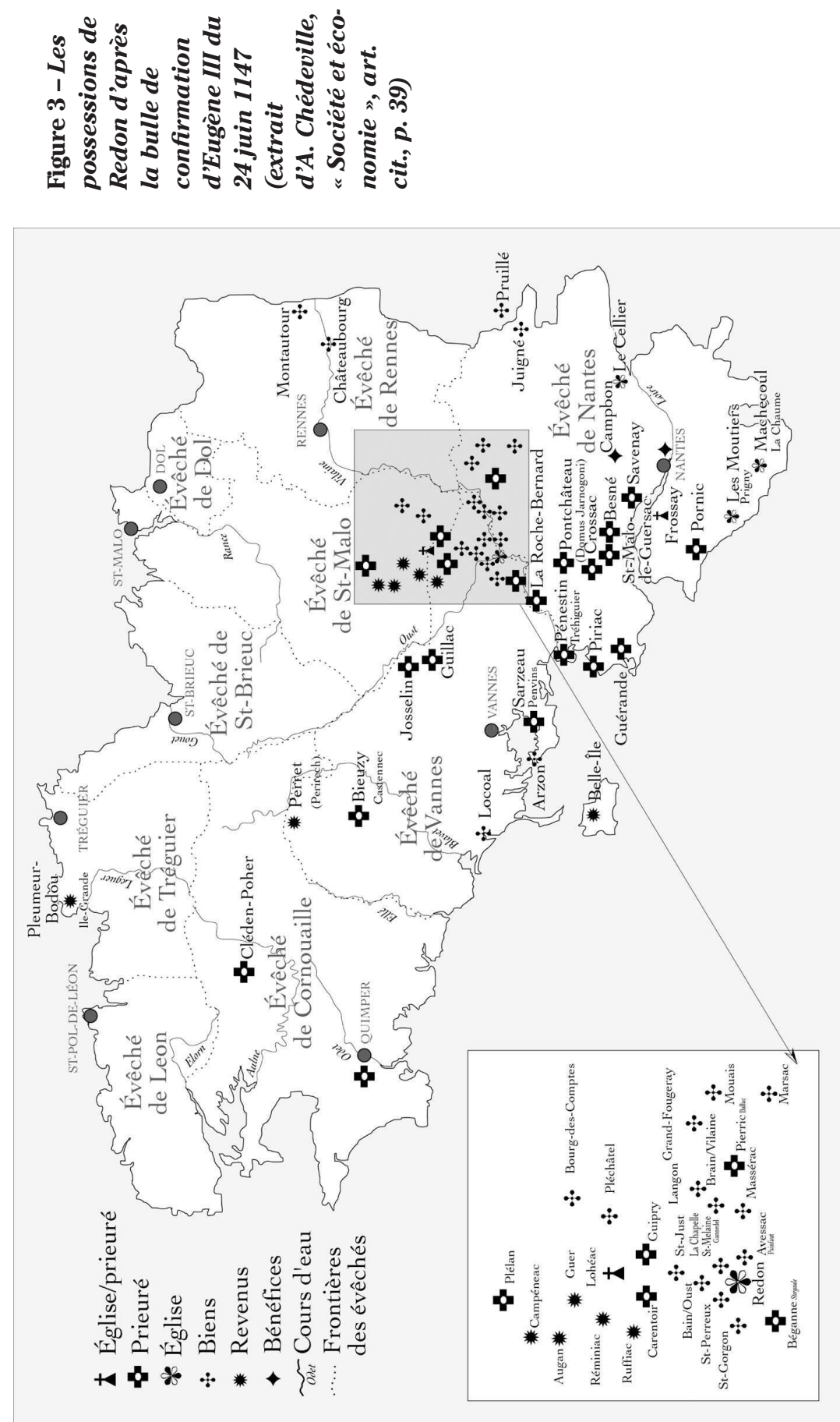
gène, couverte par le diplôme d'immunité accordé par Charles le Chauve en $850^{26}$. Il s'agissait en effet de se prémunir contre les appétits ecclésiastiques ou laïques aiguisés par l'affaiblissement de la communauté au cours du Xe siècle et de constituer pour cela, de manière classique, de solides dossiers de cartae mobilisables face à d'éventuelles calumniae, ce que suggèrent les rubriques à nette dominante topographique rédigées par les cartularistes $^{27}$. En cela les actes carolingiens apparaissent avant tout comme un instrument de consolidation patrimoniale. Certains actes des $\mathrm{XI}^{\mathrm{e}}$-XII ${ }^{\mathrm{e}}$ siècles en fournissent une attestation indirecte en renvoyant, à plus ou moins bon escient d'ailleurs, à la documentation carolingienne antérieure, à l'image des deux actes de 1021 au sujet des droits épiscopaux qui auraient été concédés à Redon par l'évêque de Vannes ${ }^{28}$.

Un second motif réside sans doute dans le désir de rappeler l'ancienneté et le prestige de l'abbaye de Redon dans un contexte où celle-ci subit le rude défi des abbayes ligériennes (Marmoutier, Saint-Florent de Saumur, SaintSerge d'Angers) et bretonnes (Saint-Melaine et Saint-Georges de Rennes, Sainte-Croix de Quimperlé). Hubert Guillotel a bien montré que les conflits seigneuriaux opposant Redon à Marmoutier et à Quimperlé expliquaient la confection de certains actes faux ou interpolés sous les abbatiats d'Hervé (1107-1134) et d'Yves (1144-v.1156-1164) ${ }^{29}$. Avec Marmoutier, le conflit se cristallisa sur la possession de Béré, disputée de 1063 à 1107/1108, et suscita à plusieurs reprises l'intervention de la papauté ${ }^{30}$. Le litige, une première fois tranché par Alexandre II en 1068, fut définitivement conclu en 1110 en faveur de Marmoutier par l'arbitrage du légat Gérard d'Angoulême qui dédommagea Redon en lui attribuant l'île d'Er en Brière ${ }^{31}$. Avec SainteCroix de Quimperlé, la nouvelle fondation des comtes de Cornouailles, le conflit portait sur Belle-Île et occupa les années 1115-1118/1119. L'affaire était confuse et le fait que le premier abbé de Sainte-Croix, vers 1047-1050, avait été moine de Redon, a sans doute compliqué les choses. Quoiqu'il en soit, l'arbitrage du légat Gérard, aux conciles d'Angoulême (1118) puis de Reims (1119), débouta de nouveau Redon pourtant soutenue par le comte

26. C'est aussi pour ces domaines que les abbés s'efforcent d'obtenir des privilèges ecclésiastiques de la part de l'évêques de Vannes ( $c f$. infra).

27. GuILlOTEL, Hubert, "Le manuscrit ", p. 17. On distingue ainsi un dossier pour Ruffiac (f $\left.{ }^{\circ} 84-92=C R, \mathrm{n}^{\circ} 138-161\right)$, Bains-sur-Oust (f $\left.{ }^{\circ} 98-99=C R, \mathrm{n}^{\circ} 181-186\right)$, Lusanger et Derval $\left(f^{\circ} 122-124=C R, \mathrm{n}^{\circ} 224-231\right) \ldots$

28. $C R, \mathrm{n}^{\circ} 321$ et 356 (probablement interpolés). Voir désormais l'édition critique de ces actes dans HENRY, Cyprien, Les actes des évêques bretons, début Xle-milieu XIf siècle, École nationale des chartes, thèse dactyl., 2010, $\mathrm{n}^{\circ} \mathrm{V} 1$, p. 1092-1097.

29. GuILlOTEL, Hubert, " Le manuscrit ", art. cit., p. 19-20.

30. Du légat Étienne, dès 1068, au concile de Bordeaux, jusqu'au légat Gérard d'Angoulême, en 1110, au concile de Nantes.

31. Sur l'action de Gérard, évêque d'Angoulême depuis 1102, nommé légat dans les provinces de Bourges, Tours, Dol, Bordeaux et Auch par Pascal II en 1108, bon connaisseur des affaires bretonnes, voir en dernier lieu : KuMAOKA Soline, "Les jugements du légat Gérard d'Angoulême en Poitou au début du XII ${ }^{\mathrm{e}}$ siècle ", Bibliothèque de l'École des chartes, 155, 1997, p. 315-338. 
Conan III ${ }^{32}$. Au début du XII ${ }^{\mathrm{e}}$ siècle, la position de Redon dans le paysage ecclésiastique de la Bretagne n'était donc pas aussi assurée qu'on pourrait le croire et se revendiquer d'une prestigieuse origine carolingienne ne pouvait que la conforter.

La composition de la Vita Conwoionis s'inscrit dans la même perspective. En célébrant la figure de l'abbé fondateur, il s'agissait à la fois d'héroïser le temps des origines et de lancer un nouveau culte spécifiquement abbatial - ce dont témoigne la division du texte en leçons liturgiques - alors que l'abbaye avait vu, au $\mathrm{x}^{\mathrm{e}}$ siècle, s'amoindrir son patrimoine de reliques ${ }^{33}$. Dans ce cadre, les retouches apportées au récit de fondation carolingien transmis par les Gesta sanctorum Rotonensium, un texte composé peu après $868^{34}$, apparaissent significatives ${ }^{35}$. La première concerne Conwoion luimême : la Vita en fait un diacre de l'évêque de Vannes apparemment en bons termes avec son évêque (et de ses compagnons des membres du clergé vannetais), quand les Gesta soulignaient au contraire les réserves de ce dernier envers le projet de fondation monastique ${ }^{36}$. Il faut certainement voir dans cette inflexion le souhait de relations apaisées avec l'évêque de Vannes dans un contexte où l'abbaye recherche et obtient de celui-ci certains privilèges ecclésiastiques (peut-être dès 1021, de manière sûre en $1093^{37}$ ) et soutient, à partir du milieu du XI ${ }^{\mathrm{e}}$ siècle, la réforme pontificale et épiscopale. L'abbaye, dont la situation aux confins de quatre diocèses (Vannes, Nantes, Alet et Rennes), le privilège d'immunité reçu de l'empereur et les relations privilégiées avec les lignées aristocratiques du Porhoët avaient sans doute favorisé une certaine autonomie, se voyait désormais

32. Sur ce conflit, voir PocqueT-Du-HAuT-Jussé, Barthélemy, Les papes et les ducs de Bretagne, essai sur les rapports du Saint-Siège avec un état, Paris, 1928, t. 1, p. 23-31 et GuILlotel, Hubert, Actes des ducs de Bretagne, op. cit., p. 135-138.

33. Au IX ${ }^{\mathrm{e}}$ siècle, les principales reliques de l'abbaye sont celles d'Apothème, volées à Angers sous l'abbatiat de Conwoion (d'après les Gesta sanctorum Rotonensium, éd. BRETT, Caroline, The Monks of Redon, op. cit., p. 170-175), de Marcellin, données par le pape à l'abbé Conwoion vers 840-847 (ibidem, p. 175-183) et de Just (CR, $\left.\mathrm{n}^{\circ} 274\right)$. Les reliques de Maixent n'ont vraisemblablement jamais été déposées à Redon mais seulement à Maixent en Plélan (où elles sont attestées peu avant $869: C R, \mathrm{n}^{\circ} 241$ ).

34. Ferdinand Lot datait le texte de 868-875 et en attribuait la paternité à Ratvili, moine de Redon et futur évêque d'Alet. Caroline Brett, sa dernière éditrice, est moins sûre de cette attribution et propose en conséquence une datation plus large, entre 868 et les environs de 919/920 (cette dernière date renvoyant à l'exil supposé des moines de Redon en Poitou, auquel il faut en réalité renoncer : $c f$. l'excursus en fin de cet article). Jean-Claude Poulin écarte lui aussi l'attribution à Ratvili et date le texte de 868-876. Dernière mise au point avec références bibliographiques : Poulın, Joseph-Claude « Le dossier hagiographique de saint Conwoion ", art. cit., et L'hagiographie bretonne, op. cit. Rappelons que le titre des Gesta n'est pas d'origine, mais qu'il est probablement dû à Mabillon.

35. Les différences entre le récit des Gesta et celui de la Vita sont recensées par BRETT, Caroline, The monks of Redon, op. cit., p. 12.

36. Vita sancti Conwoionis, éd. BRETT, Caroline, The Monks of Redon, op. cit., p. 229 : "[...] ecclesiae Venetensis diaconi arcem, exhortante Rainario eiusdem urbis pontifice, meruit conscendere."; ibidem, p. 231 : "Hunc ergo locum, paucis ex Venetensi clero secum assumptis Dei famulis, elegit expetendum."

37. Cf. infra. 
intégrée, dès les origines, au diocèse de Vannes. Son lieu de fondation était d'ailleurs clairement situé " in Venetensi territorio ${ }^{38}$ ".

La seconde inflexion de la geste des origines réalisée par la Vita réside dans la survalorisation du rôle de l'empereur Louis le Pieux aux dépens des élites régionales et des souverains bretons en particulier, qui sont ouvertement déconsidérés ${ }^{39}$. L'empereur est désormais présenté comme le fondateur de l'abbaye et l'auteur de la dotation initiale ${ }^{40}$, une affirmation reprise dans certains actes diplomatiques, telle cette notice de 1089 d'un jugement que le comte Alain IV garantit de son autorité ${ }^{41}$. Cette relecture doit être rapprochée de l'attention privilégiée accordée à la concession de l'immunité ${ }^{42}$ : dans la Vita, la dotation foncière initiale fait en effet l'objet d'une soigneuse délimitation topographique - elle est située entre deux rivières et un fundus - et se voit d'emblée pourvue d'un statut immuniste dont on sait qu'il ne fut acquis qu'une vingtaine d'années plus $\operatorname{tard}^{43}$.

Ainsi, la Vita Conwoionis opère-t-elle une réécriture des origines de Redon favorable à l'abbé, aux évêques, en particulier celui de Vannes, et au souverain carolingien, mais marginalisant le rôle des princes bretons dont les comtes des $\mathrm{XI}^{\mathrm{e}}-\mathrm{XII}{ }^{\mathrm{e}}$ siècles pouvaient se revendiquer. Cette dépréciation du rôle des élites laïques régionales est accentuée par l'engagement de l'abbaye dans la réforme " grégorienne " et la surévaluation de ses liens réels ou supposés avec la papauté.

38. Vita sancti Conwoionis, éd. BretT, Caroline, The Monks of Redon, op. cit., p. 229 : "Cum vero doctrinae insisteret, et uices pontificis diligenter exsequeretur, decursis aliquot annorum spatiis, mundi gloriam fugiens et uerae philosophiae dare operam gliscens, in Venetensi territorio solitudinis locum Rothonum nuncupatum petiit, iuxta sinum duorum nobilium fluminum situm."

39. BRETT, Caroline, The Monks of Redon, op. cit., p. 16. Le machtiern Ratvili notamment est ignoré par la Vita sancti Conwoionis. Nominoé, qui aurait apporté un appui décisif, se voit relégué au second plan derrière l'empereur.

40. Vita sancti Conwoionis, éd. BRETT, Caroline, The Monks of Redon, op. cit., p. 237 : "Cum autem Nominoius, qui intimus secretorum erat regalium, precibus instaret ut uir Dei exaudiretur, petitioni cedens imperator obtulit pro sua successorumque salute Saluatoris altari quemadmodum uir Dei petierat locum Rothonensem ab omni homine liberum et immunem, proprio confirmans donationem annulo, anno imperii sui uigesimo primo, incarnati uero uerbi octingentesimo trigesimo tertio. " En réalité, le soutien de l'empereur n'intervint qu'en 834, deux ans après la fondation de l'abbaye, peut-être en raison des réserves de Louis le Pieux envers Nominoé (qui s'était présenté comme le protecteur de la nouvelle fondation). Cf. BRETT Caroline, "L'abbaye carolingienne de Redon ", art. cit.

41. $C R, \mathrm{n}^{\circ} 290=$ Guillotel, Hubert, Actes des ducs de Bretagne, op. cit., n 99 : "Hludovicus pius Francorum Brittanorumque imperator, qui hunc Sancti Salvatoris locum a fundamento construxit."

42. L'auteur connaît les deux diplômes de Louis le Pieux de première main : cf. Poulin, Joseph-Claude, " Le dossier hagiographique de saint Conwoion ", art. cit., p. 158.

43. Vita sancti Conwoionis, éd. BRETT, Caroline, The Monks of Redon, op. cit., p. 235-237 : "Cernes denique uir Dei duotum erga se principis animum, suppliciter eum deprecatur ut illi pro aeterna remuneratione locumtribueret Rothonensem, sicut fluminibus cingitur duobus Dutulo scilicet et Undoennensi et termino Spilucensi diuiditur fundo. " L'immunité fut en réalité seulement acquise grâce au diplôme de Charles le Chauve de 850 (CR, Appendice, $\left.n^{\circ} 28\right)$. 


\section{L'engagement dans la réforme de l'Église}

Même si Redon, à la différence de l'abbaye de Marmoutier, ne constitue pas un fer-de-lance de la réforme pontificale, ses abbés ont tôt senti l'intérêt d'un rapprochement avec Rome. Dès 1050, l'abbé Pérénès, menacé de déposition, fait le voyage de Rome avec l'évêque de Rennes Main pour bénéficier avec lui d'une cérémonie de réordination exceptionnelle des mains de Léon IX ${ }^{44}$. C'est dans la foulée de cette normalisation canonique que le pape désigne Airard, cardinal de Saint-Paul-hors-les-Murs, comme évêque de Nantes en lieu et place de Budic, issu de la maison comtale, déposé en octobre 1049 au concile de Reims ${ }^{45}$. En 1060, l'évêque de Rennes Main participe au concile de Tours présidé par le cardinal Étienne ${ }^{46}$. Se dessine ainsi, de manière précoce, une Bretagne orientale et méridionale pro-pontificale regroupant les sièges de Vannes, Rennes et Nantes et au cœur de laquelle s'élève l'abbaye de Redon. En 1067, celle-ci accueille logiquement le cardinal Étienne, en tournée de légation dans l'ouest de la France ${ }^{47}$. Entre 1073 et 1084 enfin, l'abbé Aumod reçoit de Grégoire VII une bulle de protection pontificale $^{48}$. L'abbaye est alors placée " sous le droit de la sainte Église romaine ", à laquelle elle s'engage à verser un cens annuel de trois deniers d'or. Le pape confirme la libre élection de l'abbé et l'immunité jadis accordée par les souverains francs, précisant qu'aucun prélèvement d'origine séculière ne doit peser sur les domaines monastiques ${ }^{49}$. En revanche, la bulle n'accorde pas l'exemption épiscopale : l'ordination des moines-prêtres et la consécration des églises en possession de Redon devaient toujours être effectuées par le diocésain, à commencer par l'évêque de Vannes. Deux actes en faveur de l'abbé Catwallon, attribués à l'évêque de Vannes Judicaël (apr. 990-av. 1037), autre frère du comte Geoffroy I ${ }^{\mathrm{er}}$, et datés de 1021, laissent cependant entendre que les moines, qui prétendaient en avoir déjà bénéficié à l'époque carolingienne (ce qui est impossible), auraient obtenu certaines prérogatives juridictionnelles épiscopales ${ }^{50}$. La sincérité de ces actes n'est toutefois

44. Guillotel, Hubert, " Bretagne et papauté ", art. cit., p. 268-269.

45. Ibidem, p. 269.

46. Ibid., p. 273

47. Le 11 mars de la même année, il est à Saumur en compagnie des évêques de Nantes et Vannes et de l'abbé de Saint-Gildas-de-Rhuys : ibid., p. 274.

48. JAFFE 5280 (1073, après 22 avril-1084) = CR, n ${ }^{\circ} 343$ (inachevée, d'après le cartulaire de Redon) et Appendice ${ }^{\circ} 62$ (complète, d'après les deux manuscrits du Mont-Saint-Michel cité supra n. 24).

49. "Proinde juxta petitionem tuam praefato monasterio, cui tu praeesse dignosceris, et quod juris Sanctae Romanae Ecclesiae esse dignoscitur, unde per singulos annos census trium denariorum aureorum sibi redditur, hujusmodi privilegia praesenti auctoritatis nostrae decreto indulgemus, concedimus atque firmamus. [...] Hoc quoque presenti capitulo subjungimus, ut ipsum monasterium et abbates ejus vel monachi ab omni saecularis servitii sint infestatione securi, omnique gravamine mundanae oppressionis remoti, in sanctae religionis observatione seduli et quieti, nulli alii, nisi Romanae et Apostolicae Sedi, cujus juris est, aliqua teneantur occasione subjecti."

50. $C R, \mathrm{n}^{\circ} 356$ = Gullotel, Hubert, Actes des ducs de Bretagne, op. cit., $\mathrm{n}^{\circ} 12=$ HeNRY, Cyprien, Les actes des évêques bretons, op. cit., $\mathrm{n}^{\circ} \mathrm{V} 1$, rédaction $\mathrm{I} ; C R, \mathrm{n}^{\circ} 321=\mathrm{HENRY}$ Cyprien, Les actes des évêques bretons, op. cit., $\mathrm{n}^{\circ} \mathrm{V} 1$, rédaction II. Cette donation se 
pas complètement assurée, ne serait-ce que parce que la nature exacte et la portée territoriale de ces prérogatives sont énoncés curieusement et se laissent mal saisir ${ }^{51}$. S'il est fort probable qu'ils rendent compte d'une revendication monastique, sans doute exprimée dès le début du $\mathrm{XI}^{\mathrm{e}}$ siècle, de disposer d'une certaine autonomie vis-à-vis de l'évêque diocésain, il semble plus délicat d'apprécier l'ampleur de la concession épiscopale ${ }^{52}$. En tout cas, un acte de 1093 entre l'évêque Morvan et l'abbé Justin, qui ne soulève pour sa part aucun problème d'authenticité, nous apprend qu'il existait des conflits récurrents entre l'évêque et l'abbaye au sujet des églises paroissiales en possession des moines, preuve que la concession de l'évêque Judicaël, si tant est qu'elle ait eu lieu, n'était pas consensuelle. Morvan et Justin parvinrent alors à un compromis par lequel l'évêque consentait à abandonner certains de ses droits, désormais bien définis et clairement désignés par le terme episcopatus, en échange notamment de la participation régulière de l'abbé aux assemblées synodales ${ }^{53}$. À une date inconnue, les moines tentèrent d'aller plus loin en élaborant un faux privilège d'exemption attribué à l'évêque Courantguen (852-868) autorisant l'abbé à recourir à l'évêque de son choix pour l'ordination des moines ${ }^{54}$. Il ne semble pas avoir été accrédité par les évêques.

Un autre document révèle que Redon cherchait à faire remonter la protection pontificale dont elle bénéficiait avant l'époque de Grégoire VII. Une fausse bulle attribuée à Léon IX (1049-1054), seulement connue par le cartulaire, plaçait en effet l'abbaye sous la protection de « la sainte Église romaine " en des termes directement empruntés à la bulle de Grégoire VII ${ }^{55}$. Elle confirmait également à Redon la possession de Belle-Île, dont on a vu qu'elle lui était disputée par Sainte-Croix de Quimperlé. Cette confirmation

présente comme la restitution d'un droit octroyé par les évêques Susan (841-848) et Coranjenus (852-868), ce qui est absolument impossible, aucun évêque carolingien n'ayant jamais octroyé de tels droits à des moines.

51. Ces actes sont considérés comme interpolés par Hubert Guillotel. Mais sur le fond, ils sont en grande partie réhabilités par Cyprien Henry. Il n'en demeure pas moins que la formulation de ces droits (presulatum ou ius presulatus) reste tout à fait singulière et que le territoire où ils s'appliqueraient reste vague. Il ne saurait s'agir de l'ensemble du diocèse de Vannes puisqu'il ne s'agit en aucune manière d'un privilège d'exemption.

52. Il reste que s'il s'agit d'une interpolation, celle-ci reste difficile à dater : elle peut remonter à la première moitié du XI ${ }^{\mathrm{e}}$ siècle (le terme archidiaconatum, donné comme équivalent de presulatum, se répand à partir de cette époque); elle peut être liée à l'acquisition de la bulle de Grégoire VII ; elle peut enfin être postérieure à celle-ci (mais antérieure à 1093 : cf. infra et note suivante).

53. HENRY, Cyprien, Les actes des évêques bretons, op. cit., $\mathrm{n}^{\circ} \mathrm{V} 4$ : l'arbitrage porte sur la participation de l'abbé et des prêtres au synode diocésain, sur l'exercice par l'abbé de la justice canonique sur les prêtres et les desservants, enfin sur le droit de visite de l'évêque à Redon.

54. $C R$, Appendice $n^{\circ} 46$ (cette forgerie est peut-être en relation avec les événements de 1127 : cf. infra).

55. JAFFE 4159 (13 avril, sans doute 1049) = CR, n 378 : l'abbaye est placée sour le ius Sancte Romane Ecclesie en échange d'un cens recognitif annuel de trois deniers d'or. Cette bulle est adressée à l'abbé Catwallon alors que celui-ci est décédé le 16 janvier 1041 . 
venait conforter une fausse notice datée du 22 mars 1026 détaillant les circonstances d'une pseudo-donation du comte Geoffroy ${ }^{\mathrm{er}}$ en faveur de Redon ${ }^{56}$. Comme Hubert Guillotel l'a montré, ces deux actes ont probablement été fabriqués sous l'abbatiat d'Hervé, vers 1115-1116, pour contrer l'abbaye rivale ${ }^{57}$. Du même coup, les moines de Redon prétendaient bénéficier de la protection romaine depuis le milieu du XI ${ }^{\mathrm{e}}$ siècle, une époque où aucune autre abbaye bretonne n'en disposait, à commencer par Quimperlé qui avait reçu une bulle de Grégoire VII en $1078^{58}$.

La Vita Conwoionis fait remonter les liens privilégiés entre Redon et la papauté bien plus avant encore. Elle rappelle bien sûr, à la suite des Gestes des saints de Redon, le voyage accompli par Conwoion à Rome, d'où l'abbé était revenu chargé des reliques de saint Marcellin, pape et martyr, qui allaient transformer Redon en lieu de miracles et en mémorial pontifical ${ }^{59}$. Mais la Vita modifie surtout le récit de fondation en associant le pape à l'une des donations de l'empereur Louis. L'hagiographe précise en effet que l'empereur aurait donné à Redon non seulement les cinq vici de Bains, Langon, Renac, Brains et Arzon, mais également leurs églises qui lui auraient été préalablement données par le pontife romain lui-même, avec l'accord de l'évêque Rainald de Vannes ${ }^{60}$. Ce récit à l'évidence anachronique, qui entend légitimer la possession d'églises par un laïc, fût-ce l'empereur, s'explique d'abord par le contexte réformateur dans lequel la Vita fut composée. Mais du même coup il associe la papauté aux premiers temps de l'abbaye.

Un dernier document de la deuxième moitié du XI ${ }^{\mathrm{e}}$ siècle cherche à enraciner la relation entre la papauté et Redon dans l'époque carolingienne tout en "romanisant " le trésor des reliques de l'abbaye. Les moines ont en effet entièrement forgé, probablement au moment de la mise en œuvre du cartulaire ou peu avant, une fausse bulle adressée au " duc de Bretagne " Salomon par le pape Adrien II (vers 867-874) attestant la donation à l'abbaye de reliques de Léon III, le pape qui couronna Charlemagne empereur, et instituant à Redon un pèlerinage susceptible de remplacer le voyage de Rome ${ }^{61}$. Cette dernière clause fut d'ailleurs reprise dans un autre acte suspect (une notice datée de 1047) à l'occasion de sa transcription dans le cartulaire au début du $\mathrm{XII}^{\mathrm{e}}$ siècle, indice de son importance aux yeux

56. $C R, \mathrm{n}^{\circ} 296$ = Gullotel, Hubert, Actes des ducs de Bretagne, op. cit., $\mathrm{n}^{\circ} 14=$ HeNRY, Cyprien, Les actes des évêques bretons, op. cit., $\mathrm{n}^{\circ} \mathrm{V} 1^{*}$.

57. Gulllotel, Hubert, Actes des ducs de Bretagne, op. cit., p. 135-136 et 182-183.

58. Quimperlé avait obtenu un privilège de Grégoire VII le 25 mars 1078 (JAFFÉ 5072 : Cartulaire de l'abbaye Sainte-Croix de Quimperlé, op. cit. p. 132).

59. Gesta sanctorum Rotonensium, éd. BRETT Caroline, The Monks of Redon, op. cit., § 9-10.

60. Vita sancti Conwoionis, éd. BRETT Caroline, The Monks of Redon, op. cit., p. 239 : "Ecclesias etiam earumdem plebium, quas ipse imperator Romani pontificis munere obtinebat, simili iure praefato tribuit abbati, adsistente regis praesentiae Rainaldo Venetensis ecclesiae praesule, et idipsum sigillo suae munitionis corroborante."

61 . JAFFE $2950=C R, \mathrm{n}^{\circ} 90$. D'après ce faux privilège, le fait de se rendre trois fois en pèlerinage à Redon dispense du vœu d'aller à Rome. 
des moines ${ }^{62}$. Le trésor abbatial s'enrichissait ainsi de nouvelles reliques pontificales, qui plus est étroitement liées au souvenir du prestigieux empereur franc, et Redon était érigée en petite Rome pour " les habitants de Bretagne ", ainsi que l'énonçait la fausse bulle d'Adrien II ${ }^{63}$.

Si les moines de Redon exagèrent ainsi l'ancienneté et l'étroitesse de leurs relations avec la papauté, ils souhaitent également souligner leur soutien à la réforme épiscopale, à l'image de la plupart des abbayes réformatrices de l'ouest de la France. En témoignent pour le diocèse de Vannes, en dépit des quelques tensions évoquées ci-dessus, le nouveau récit des origines promu par la Vita Conwoionis et l'accord de 1093 au sujet des droits épiscopaux sur les églises dépendantes de l'abbaye. L'abbé Aumod avait par ailleurs consenti, dès 1062, au versement du cens épiscopal exigé par l'évêque de Nantes Quiriac, le successeur d'Airard, pour toutes les églises de son diocèse ${ }^{64}$.

Au-delà de ces relations privilégiées avec la papauté, largement fantasmées, ou de ces liens plus concrets avec les évêques réformateurs, le signe le plus net de l'engagement de l'abbaye dans la réforme réside toutefois dans le discours à forte tonalité grégorienne dont témoignent les notices rédigées dans la deuxième moitié du $\mathrm{XI}^{\mathrm{e}}$ siècle ou au début du $\mathrm{XII}^{\mathrm{e}}$ siècle. Deux exemples permettent d'en prendre la mesure. Le premier, nous le trouvons dans la notice racontant la donation, en 1047-1048, de l'église SainteMarie de Montautour par le prêtre Raoul, puis sa confirmation par Conan II " le jour où il fut fait comte ${ }^{65}$ ». Le récit glose notamment deux citations évangéliques de façon tout à fait significative. Une première citation - " celui qui laisse son père ou sa mère ou sa femme ou ses fils ou ses champs à cause du royaume de Dieu recevra le centuple [de ce qu'il a abandonné] et possèdera la vie éternelle " (Matthieu 9, 29) - vient justifier le choix du donateur de transmettre ses biens à Redon aux dépens de sa famille; elle fait jouer l'opposition entre liens spirituels et liens charnels et souligne la supériorité des premiers sur les seconds. La deuxième citation - " il faut rendre à César ce qui est à César et à Dieu ce qui est à Dieu " (Matthieu 22, 21) -, mise dans la bouche de l'évêque de Rennes Main (av.1047-†1076), sert à légitimer la distinction entre biens séculiers (secularia) et biens ecclésiastiques (aecclesiastica) pour mieux fonder le transfert de l'église, des droits paroissiaux et des domaines qui lui sont attachés entre les mains des

62. Voir à ce sujet Guillotel, Hubert, Actes des ducs de Bretagne, op. cit., p. 294-296.

63. Cf. la formule d'envoi de la bulle : "Valete in Christo fideles Britanniae habitatores, amen."

64. $C R, \mathrm{n}^{\circ} 285$ et 362 . Voir GuILLOTEL, Hubert, " La pratique du cens épiscopal ", art. cit. p. $21-22$.

65. $C R, \mathrm{n}^{\circ} 294$ : " in die quo chomes factus est ". Sur cette notice, voir également : PICHOT Daniel, "La notice 294 du Cartulaire de Redon, les moines, la réforme et la société ", dans Qhaguebeur, Joelle, Soleil, Sylvain (dir.), Le pouvoir et la foi au Moyen Âge en Bretagne et dans l'Europe de l'Ouest. Mélanges en mémoire du professeur Hubert Guillotel, Rennes, 2010, p. 131-143, qui en propose une traduction française. 
moines $^{66}$. La possession par les laïcs de res ecclesiasticae - les lieux de culte et les droits qui leur étaient associés, qu'ils soient liés à la parrochia ou simplement à la dotation foncière de l'église - est ainsi déligitimée. Tout juste les laïcs, en l'occurrence les seigneurs de Vitré, se voient-ils reconnaître un droit de custodia, moyennant le versement d'un cens annuel de 5 sous.

Une autre notice du début du $\mathrm{XII}^{\mathrm{e}}$ siècle raconte la fondation par Guéthénoc, "vicechomes de castello Thro ${ }^{67}$ ", à une date inconnue, d'un nouveau château, dont il promet de remettre la chapelle aux moines de Redon - bien que la notice ne l'explicite pas, il s'agit à l'évidence, comme prend soin de le préciser la rubrique du cartulariste, du château de Josselin ${ }^{68}$. Ce récit revêt une triple finalité. Il vise d'abord à attester que Redon était en possession de la chapelle castrale de Josselin, opportunément dédiée au Sauveur, depuis les origines (Guéthénoc était l'arrière-grand-père du vicomte Josselin II [ $† 1114]$ ), vraisemblablement pour contrer les moines de Marmoutier, implantés à Saint-Martin de Josselin à partir de $1105^{69}$. Ensuite, en soulignant la valeur d'un autre don effectué par Guéthénoc qui offrit également aux moines de Redon une table d'argent dorée pour l'autel de l'abbatiale, en recourant pour le désigner à une titulature emphatique (venerabilis proconsul), puis en affirmant que son élection de sépulture dans l'abbaye relevait d'une coutume générale au sein de la noblesse ${ }^{70}$, la notice entend témoigner de la vigueur du lien à la fois spirituel et social unissant la communauté de Redon à la lignée des vicomtes de RennesPorhoët et plus largement à l'aristocratie bretonne. Enfin, en évoquant le rôle peu vraisemblable qu'auraient joué les moines dans le rituel de fondation du château et de la chapelle - ceux-ci auraient été appelés à en choisir l'emplacement et à assister à la plantation symbolique d'un pieu, intervenue un dimanche ${ }^{71}-$, et surtout en mentionnant l'institution d'un cens recognitif de 5 sous à verser chaque année à l'abbaye, la notice place le château lui-même, et non seulement sa chapelle, sous le dominium direct de l'abbaye : castellum... ecclesie Rothonensi subjugavit n'hésite pas à dire le texte ${ }^{72}$. Au-delà de la célébration convenue de l'amicitia entre moines et

66. CR, $\mathrm{n}^{\circ} 294$ : « [...] aecclesiam sancte Mari $₫$ de Montaltor, cum omni parrochia sua, cum terris, silvis, pascuis, aquarumque decursibus. "

67. Sur ce personnage et sa famille, voir GuILLOTEL, Hubert, « De la vicomté de Rennes à la vicomté de Porhoët (fin du X ${ }^{\mathrm{e}}$-milieu du XII ${ }^{\mathrm{e}}$ siècle) ", Mémoires de la Société d'histoire et d'archéologie de Bretagne, 73, 1995, p. 5-23.

68. $C R, \mathrm{n}^{\circ} 292$ (la rubrique indique : "De castello Goscelini "). Sur le caractère falsifié de cette notice (titulatures anachroniques, modalités et mise en scène de la fondation improbables...), voir Guillotel, Hubert, « De la vicomté de Rennes », art. cit., p. 16-17.

69. Guillotel, Hubert, " De la vicomté de Rennes ", art. cit., p. 19-20.

70. $C R, \mathrm{n}^{\circ} 292:$ : [...] peciitque ab eis corpus suum, dum vita excederet, et heredum suorum, sicut mos erat Britannie nobilium, in eodem loco sepeliri ".

71. Sur ce rituel, voir CHÉDEVILLE, André, «Figens palum in castello edificando, ut mos est... À propos de la fondation du château de Josselin ", dans ; Le pouvoir et la foi au Moyen Âge, op. cit., p. 451-458.

72. $C R, \mathrm{n}^{\circ} 292$ : "Castellum etiam aedificandum Christo Domino et ejus aecclesiae Rothonensi subjugavit, et censum, id est, quinque solidos unoquoquo anno, monachis de eo reddi constitutit". 
grands, le discours monastique donne ici à voir une véritable hiérarchie à la fois religieuse, sociale et seigneuriale.

\section{Une vision ambiguë du rôle des ducs dans le renouveau monastique}

Quelques actes du cartulaire nous renseignent sur le soutien apporté par les ducs à la communauté de Redon à partir de la fin du $x^{\mathrm{e}}$ siècle, qu'il s'agisse de la confirmation de donations effectuées par d'autres ou plus rarement de concessions personnelles. Mais si l'on écarte les actes faux ou interpolés de 958-960, 1026, 1027 et 1049 concernant les donations des îles d'Enesmur, de Locoal et de Belle-Île, par lesquels les moines de Redon s'efforcent de compenser l'engagement des nouveaux ducs issus de la maison de Cornouaille auprès de leur nouvelle fondation de Quimperlé, ce soutien reste peu mis en valeur ${ }^{73}$.

La composition du cartulaire confirme cette réserve à l'égard du rôle des ducs dans le renouveau abbatial. Le cartulaire s'ouvre en effet, comme nous l'avons vu, sur les actes fondateurs carolingiens et ne comporte aucun dossier spécifique rassemblant les actes ducaux, ni en ouverture, ni après. Lorsque le travail de compilation est repris sous l'abbatiat de Robert (10861091), le premier acte copié est un acte de 1089 rapportant un conflit entre les chapelains du comte et les moines de Redon donnant l'occasion à ces derniers de rappeler la fondation de leur abbaye par " Louis le Pieux, empereur des Francs et des Bretons " ${ }^{74}$. Les copies d'actes ducaux se situent toutes dans les additions postérieures de la fin $\mathrm{du} \mathrm{XI}^{\mathrm{e}}$ ou du XII ${ }^{\mathrm{e}}$ siècle et y figurent en ordre dispersé.

Pourtant, il paraît évident que la maison de Rennes joua un rôle décisif dans le renouveau de l'abbaye de Redon à partir de la fin du $x^{\mathrm{e}}$ siècle. $\mathrm{Si}$, dans le contexte général de la réforme monastique, les ducs de Bretagne s'adressèrent de préférence, comme les autres princes de l'ouest de la Francie, à Fleury ou Marmoutier pour réformer ou refonder d'anciens établissements tombés en déshérence, ils considéraient semble-t-il Redon, probablement en raison de son passé carolingien, comme l'abbaye la plus importante de leur principauté. Incite notamment à le penser le fait que les deux abbés en fonction dans la première moitié du XI ${ }^{\mathrm{e}}$ siècle $^{75}$, étaient, comme nous l'avons

73. Les actes ducaux sont les suivants : confirmation de Conan $\mathrm{I}^{\mathrm{er}}$ (v. 981-992) (CR, n 357 = Guillotel Hubert, Actes des ducs de Bretagne, op. cit., n ${ }^{\circ} 7$ ); donations (1019, v. 1008-1040) ou confirmations (1009/1019-1040, 1021) d'Alain III (CR, ${ }^{\circ} 323,299,289,321$ = ibid., $\left.\mathrm{n}^{\circ} 11,37,38,12\right)$; confimations de Conan II (1047, v. 1061-1066) $\left(C R, \mathrm{n}^{\circ} 294\right.$ et 61 = ibid., $\mathrm{n}^{\circ} 53$ et 64$)$.

74. $C R, n^{\circ} 290$ : "Hludovicus pius Francorum Brittannorumque imperator".

75. La Vita Gauzlini abbatis Floriacensis monasterii, composée par André de Fleury vers 1042, évoque un moine de Fleury nommé Teudo, venu en Bretagne aux côtés du moine Félix et qui serait devenu abbé du monastère de "Redonicum " (André de Fleury, Vie de Gauzlin, abbé de Fleury, livre 1, c. 24, éd. et trad. Robert-Henri BAUTIER et Gillette LABORIT, Paris, 1969, p. 64-67). Comme l'on sait, grâce à la Vie de saint Gildas, que Félix fut envoyé par l'abbé Gauzlin pour réformer Saint-Gildas de Rhuys et Lochemenech à la demande du duc Geoffroy I ${ }^{\text {er }}$ en 1008 (éd. Du Bors Jean, Floriacensi vetus bibliotheca..., Lyon, 1605, 
vu, étroitement liés aux comtes de Rennes, auxquels ils devaient certainement leur charge, qu'il s'agisse de Mainard, attesté à la tête de Redon d'avant 1008 jusqu'avant 1019, un ancien moine de Saint-Pierre de Gand qui fut aussi l'abbé du Mont-Saint-Michel jusqu'en $1009^{76}$, à une époque où l'abbaye était encore sous influence bretonne - le duc Conan $\mathrm{I}^{\mathrm{er}}$ fut inhumé au Mont en 992, ainsi, peut-être, que son fils Geoffroy $\mathrm{I}^{\mathrm{er}}$ en $1008^{77}$-, ou bien de Catwalon, abbé d'avant 1019 jusqu'en 1041, qui était le propre frère de Geoffroy ${ }^{\text {er }}$ et de l'évêque de Vannes Judicaël, et donc l'oncle d'Alain III ${ }^{78}$. Si l'on en juge par la nécessité où se trouva l'abbé Pérénès (1041-†1062) de faire confirmer son élection par le pape Léon IX en 1050, on peut également présumer qu'il devait celle-ci, jusque-là peu canonique, au comte de Rennes. Entre la fin du $\mathrm{X}^{\mathrm{e}}$ siècle et le milieu du XI ${ }^{\mathrm{e}}$ siècle, les destinées de l'abbaye se trouvaient donc entre les mains de la maison ducale.

Cette période et plus précisément les abbatiats de Catwallon (1009/1019$\uparrow 1041)$ et Pérénès (1041-†1062) coïncident avec le début du flux de donations issues de l'aristocratie seigneuriale, qui se poursuit de manière à peu près égale jusqu'aux années 1120-1140 (fig. 4 et 5). C'est de ces années que datent les principales donations des lignées seigneuriales pour lesquelles le modèle ducal, jusque dans le Nantais, dût pleinement jouer, telles les DolCombourg, les vicomtes de Porhoët, les seigneurs de Vitré, Châteaubriant, La Roche (-Bernard), Begon, Savenay, Migron, Ancenis ou Retz ${ }^{79}$. C'est éga-

$\S 21-23$, p. 452-456), on en a déduit qu'il en avait certainement été de même pour Teudo et l'on a parfois identifié Redonicum avec Redon, faisant de l'abbaye un nouvel exemple de communauté réformée par Fleury ( $c f$. par exemple LA LANDE DE CALAN, Charles de, "Les abbés Félix de Ruis et Teudo de Redon ", Revue de Bretagne, 40, 1908, p. 32-35). On n'a cependant aucune trace de ce Teudo dans les sources redonnaises ou bretonnes en général et son supposé abbatiat en 1008 n'est pas compatible avec la succession abbatiale telle qu'on peut la reconstituer (l'abbatiat de Mainard, dont le début ne peut être daté que de manière imprécise, a toutefois nécessairement commencé du vivant de Geoffroy I ${ }^{\text {er }}$, soit avant 1008). En outre, il semble plus probable, en dépit d'une graphie peu courante, que Redonicum renvoie ici à Rennes et donc à l'abbaye Saint-Melaine (André de Fleury, Vie de Gauzlin..., éd. cit., note 4, p. 65).

76. Cf. supra note 8.

77. Voir l'analyse de BouEt Pierre, « Le Mont Saint-Michel entre Bretagne et Normandie de 960 à 1060 ", dans : QuAGHEBEuR, Joelle, MERDRIGNAC, Bernard (dir.), Bretons et Normands au Moyen Âge. Rivalités, malentendus, convergences, Rennes, 2008, p. 165-199, ici p. 186187, en dépit d'une conception peu vraisemblable de l'indépendance des abbés du Mont vis-à-vis des pouvoirs princiers environnant entre 966 et 1008 .

78. De manière significative, les moines se sont d'ailleurs souciés d'introduire une confirmation d'Alain III au bas de l'acte suspect de 1021 par lequel Judicaël aurait concédé à l'abbé certains privilèges juridictionnels (cf. supra note 50).

79. La plupart de ces désignations sont anachroniques, mais elles permettent d'identifier facilement les principales familles : Begon : $C R, \mathrm{n}^{\circ} 327$; Dol-Combourg : $C R, \mathrm{n}^{\circ} 289$; Cellier/Ancenis : $C R, \mathrm{n}^{\circ} 303$; Châteaubriant : $C R, \mathrm{n}^{\circ}$ 287-288, 302; La Roche : $C R, \mathrm{n}^{\circ} 307$; Migron : $C R, \mathrm{n}^{\circ} 297,315,316,317,385$; Porhoët : $C R, \mathrm{n}^{\circ} 292,293$; Retz : $C R, \mathrm{n}^{\circ} 312$; Savenay: $C R$, Appendice $n^{\circ} 58$ et 59 ; Vitré : $C R, \mathrm{n}^{\circ} 294$. Sur certaines de ces familles, voir TONNERRE Noël-Yves, Naissance de la Bretagne. Géographie historique et structures sociales de la Bretagne méridionale (Nantais et Vannetais) de la fin du VIII à la fin du XII siècle, Angers, 1994, p. 326-335, 339-344, 346-348, 356-357. 
lement dans ces années que Redon commence à se voir confier en grand nombre des églises, simples chapelles ou églises paroissiales, dont certaines, comme celle de Frossay, sont alors tenues pour d'anciens petits monastères en déshérence ${ }^{80}$. Dès cette époque, le rayonnement de l'abbaye se fait sentir bien au-delà des environs de Redon, sur les diocèses de Vannes, Nantes, Alet, Rennes et Dol.

Après un $\mathrm{X}^{\mathrm{e}}$ siècle difficile marqué par un évident repli, la première moitié du $\mathrm{XI}^{\mathrm{e}}$ siècle apparaît donc comme le moment d'une véritable restauration monastique menée à l'initiative des ducs de la maison de Rennes, avec le soutien des réseaux aristocratiques qui leur sont proches. Comme ailleurs, cette restauration repose sur le tissage de liens socio-religieux étroits entre moines et grands, autrement dit sur une amicitia fondée sur la pratique du don-échange, l'offrande de biens matériels favorisant l'essor de la seigneurie monastique et permettant aux donateurs d'être associés aux bénéfices spirituels des prières des moines, une association parfois formalisée par l'intégration dans la societas et fraternitas monastique ou menée jusqu'à la conversion ad succurendum (figures 4 et 5).

Ce rôle des ducs, les compilateurs du cartulaire de Redon des années 1070-1140 choisissent soit de le minorer, soit de le remodeler, car il s'agit pour eux d'inscrire la renaissance de leur abbaye dans la prolongation d'un âge d'or carolingien profondément réinterprêté et dans une dynamique avant tout ecclésiastique dominée par la papauté et les relations avec les évêques (Vannes et Nantes surtout). Les ducs, qui entendaient également profiter du souvenir carolingien, ne leur en ont apparemment pas tenu rigueur. Peut-être parce qu'ils sont désormais, à partir d'Hoël (1066-1084), issus de la maison de Cornouaille qui avait d'autres attaches et d'autres horizons? Quoi qu'il en soit, l'un d'entre eux, Alain IV, a peut-être fait retraite et élu sépulture dans l'abbaye en 1116 ou peu après ${ }^{81}$, et Redon continue d'apparaître dans la première moitié du XII ${ }^{\mathrm{e}}$ siècle comme un éminent lieu de rassemblement non seulement du clergé breton, qui s'y réunit en 1122 et 1135-1136, mais aussi de la cour ducale : on a en effet connaissance d'assemblées en 1108, 1118 et 1127 et un acte de 1105-1107 laisse même entendre qu'il s'agissait d'une coutume ${ }^{82}$.

80. $C R, \mathrm{n}^{\circ} 315$.

81. Ceci est généralement déduit d'un acte de donation d'Alain IV daté de 1112 et passé à Redon $\left(C R, \mathrm{n}^{\circ} 370\right.$ = Gullotel Hubert, Actes des ducs de Bretagne, op. cit., $\left.\mathrm{n}^{\circ} 115\right)$. Cet acte, connu par deux rédactions différentes, soulève cependant quelques difficultés : la liste des témoins est incompatible avec la date de 1112; plus ennuyeux, seule la seconde rédaction précise qu'Alain IV, fatigué et malade, avait transmis le pouvoir ducal à son fils Conan III pour se convertir et entrer au monastère de Redon. Or nous savons par ailleurs qu'Alain régna conjointement avec Conan jusqu'en 1115 au moins (le premier acte où ce dernier intervient comme seul duc de Bretagne date de 1116). Voir la discussion d'Hubert Guillotel (ibidem, p. 423-424).

82. Guillotel, Hubert, Actes des ducs de Bretagne, op. cit., p. 413 (1108), 446-447 (1118), 455 (1127); CR, n 377 (1105-1107) : " Congregata apud Rothonum, ut moris erat, coram Alano principe, curia omnium nobilium Britannie..." 
Figure 4 - Donations et transferts de biens en faveur de Redon d'après les deux cartulaires (par abbatiat) (NB : 14 actes ne peuvent être attribués précisément à un abbatiat)

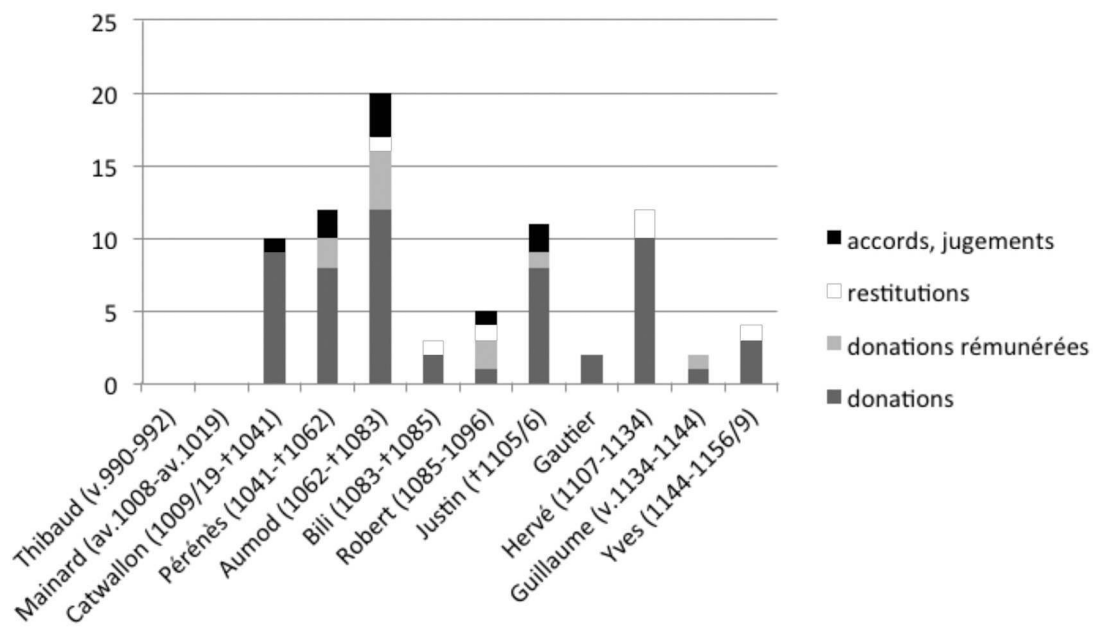

Figure 5 - Donations et transferts de biens en faveur de Redon d'après les deux cartulaires (par tranches chronologiques de vingt ans)

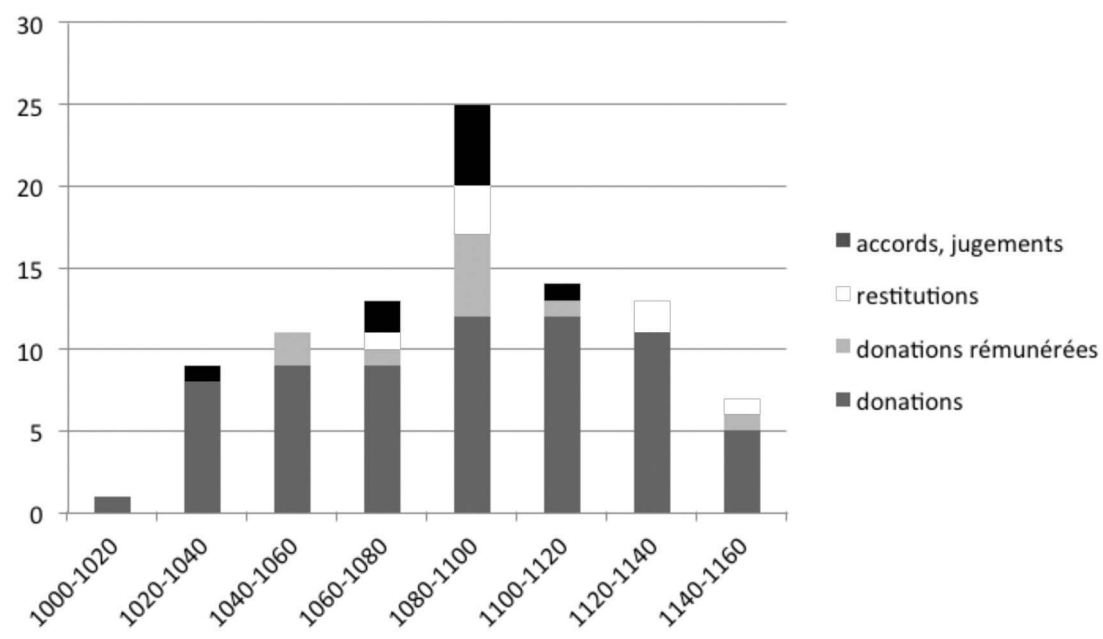




\section{Le rêve de Redon : l'abbaye, foyer des relations entre le pape et le prince et capitale religieuse de la Bretagne}

Un document exceptionnel issu du cartulaire donne à voir la place que l'abbaye estimait devoir occuper dans l'Église et dans la principauté bretonnes au milieu du XII ${ }^{\mathrm{e}}$ siècle, à la veille des déboires de l'abbé Yves. De nouveau, il s'agit d'une notice composite mêlant récit et copie de documents antérieurs, éléments sincères et fallacieux ${ }^{83}$. Elle est datée du 23 octobre 1127 et commence par une lettre apocryphe du duc Conan III au pape Honorius III par laquelle le duc remet au pape la custodia de l'abbaye, qu'il déclare ne plus parvenir à assurer face aux " perfidies accumulées des Bretons $^{84}$ ". La notice se poursuit par le récit de l'ambassade, menée par l'abbé de Redon Hervé et le prieur de Saint-Melaine de Rennes Guillaume, apportant cette missive au pape dans son palais du Latran. À Rome, après avoir déclaré conserver cette lettre dans ses archives, le pape confie au légat Gérard d'Angoulême, à l'archevêque de Tours Hildebert de Lavardin et à tous les évêques de Bretagne la charge de régler le contentieux et de reconsacrer le sanctuaire abbatial souillé par les malefactores ${ }^{85}$. Vient ensuite l'évocation de la fameuse cérémonie de reconsécration en présence du duc, de sa mère, de tous les optimates de Bretagne et d'une grande foule.

Si une cérémonie de consécration a vraisemblablement eu lieu en $1127^{86}$, nombre d'éléments de ce récit relèvent de la forgerie ou de l'interpolation. Il n'en reste pas moins très significatif de l'idéologie monastique et des prétentions de Redon. On reconnaît d'abord la rhétorique classique dénonçant les "méchants " laïcs (les malefactores), dont le principal crime (perfidia) est de s'être attaqué aux biens du monastère et d'être allé jusqu'à profaner l'église abbatiale ${ }^{87}$. Cette rhétorique entre en résonnance avec les actes dénonçant les "intolérables " ou " injustes coutumes " et encou-

83. $C R, \mathrm{n}^{\circ} 347$ = GuILlotel, Hubert, Actes des ducs de Bretagne, op. cit., $\mathrm{n}^{\circ} 131$.

84. Ibidem : "[...] accumulata Britanorum perfidia... " Il est difficile de savoir à quoi renvoie exactement cette accusation. Un acte suspect du cartulaire $\left(C R, \mathrm{n}^{\circ} 348\right)$ et deux chartes aujourd'hui perdues, seulement connues grâce à leur édition par Dom Morice ( $C R$, Appendice $n^{\circ} 70$ et 72), mentionnent les déprédations d'Olivier de Pontchâteau au lieu-dit Ballac, dans la paroisse de Pirric. Celui-ci aurait finalement accepté de faire pénitence et de céder ce lieu, ainsi que la terre appelée Brengoen, aux moines de Redon. Plusieurs autres chartes montrent que les moines ont acquis en ces lieux les droits d'autres petits seigneurs ou chevaliers dans les années 1128-1140 (CR, $\mathrm{n}^{\circ} 386,387$, Appendice $\mathrm{n}^{\circ} 71,73$, $74,75)$.

85. $C R, \mathrm{n}^{\circ} 347$ = GuILlotel Hubert, Actes des ducs de Bretagne, op. cit., $\mathrm{n}^{\circ} 131$ : " [...] et qui essent malefactores quodque malefactum et qua causa accidisset ab abbate inquisivit. "

86. Elle est mentionnée dans l'une des chartes évoquées note précédente : $C R$, Appendice $n^{\circ} 70$, malheureusement probablement en partie interpolée elle aussi (cf. GuILlotel, Hubert, Actes des ducs de Bretagne, op. cit., p. 455).

87. $C R, \mathrm{n}^{\circ} 347$ = GuILlotel Hubert, Actes des ducs de Bretagne, op. cit., $\mathrm{n}^{\circ} 131$ : « [...] ut episcopali severitate tam diu malefactores choercerent, donec aecclesie cuncta damna et quae ei male ablata fuerant, tam possessionum quam ceterarum rerum, restituerentur [...] cum summa veneratione, consecraverunt aecclesiamque ab immundicia quam obsessi in ea fecerant, purificaverunt. " 
rageant les abandons ou les restitutions de droits jugés mal acquis, dont le cartulaire montre qu'ils se multiplient à partir des années 1060 (fig. 5) ${ }^{88}$.

Derrière ce type de discours se devine un conflit seigneurial remettant en cause l'inviolabilité de la seigneurie d'Église telle que la concevaient les moines, les laïcs ayant enfreint le privilège d'immunité protégeant leurs terres et ignoré le caractère sacré du lieu saint. Le jugement du légat et la réparation des torts (definitio iniqua altercatio) doivent donc s'accompagner de la réaffirmation du statut de Redon et d'une recharge de sacralité. La notice rappelle donc que l'abbaye est placée " sous le droit de saint Pierre " depuis le pontificat de Léon IX - elle fait donc référence à la fausse bulle attribuée à Léon IX plutôt qu'à la bulle authentique de Grégoire VII -, en mettant ce rappel dans la bouche du duc Conan III ${ }^{89}$, et que les évêques de Vannes et de Nantes ont cédé à l'abbé leurs droits d'archidiaconat. En évoquant le rituel de reconsécration, elle insiste par ailleurs sur la dédicace des autels au Sauveur et aux saints romains (Pierre, Paul et Marcellin, pape et martyr) ${ }^{90}$ : Redon se donne à voir comme une petite Rome.

Tous ces éléments s'inscrivent dans la continuité des stratégies discursives mises en œuvre depuis l'abbatiat d'Aumod. Deux éléments sont plus originaux. En premier lieu, le duc et ses prédécesseurs sont présentés comme les gardiens de l'abbaye au nom du pape. C'est à ce titre que le duc se déclare même fidèle du pape : " ego et antecessores mei in fidelitate vestra hactenus custodivimus ". On sait que la custodia représente au XII ${ }^{\mathrm{e}}$ siècle une sorte de codification et d'atténuation de la protection traditionnelle exercée par les laïcs, parfois dans le cadre formel d'une avouerie, sur les établissements ecclésiastiques, une protection qui entraînait souvent divers prélèvements sur les biens d'Église, vivement dénoncés par les grégoriens. En l'occurrence, cette custodia permet aux ducs de continuer de jouer leur rôle de protecteurs de l'Église en Bretagne et de l'abbaye de Redon en particulier, mais dans un cadre canonique précis que les moines entendent défendre. La déclaration de fidelitas mise dans la bouche du duc l'engage plus encore : elle suppose, sinon forcément une entrée en vassalité (aucune allusion n'est faite à un serment ni à un quelconque service, même limité au versement d'un cens), du moins de reconnaître le pape comme son seigneur - certes non pour sa principauté mais pour l'abbaye de Redon. On

88. Quelques évocations de mauvaises coutumes : $C R, \mathrm{n}^{\circ} 312(1055), \mathrm{n}^{\circ} 316$ (vers 1041/1061-1062/1084 : "rapina »), 341 (1108 : "injustam quandam consuetudinem "), 358 (1085-1096 : "invasio ").

89. $C R, \mathrm{n}^{\circ} 347$ = GuILlotel Hubert, Actes des ducs de Bretagne, op. cit., $\mathrm{n}^{\circ} 131$ : « [...] proprio juri beati Petri sub sancto Leone papa sociavit..." "

90. Ibidem : " [...] altare in honore Salvatoris mundi sueque genitricis et beatorum Petri et Pauli sanctique Marcellini pape et martiris atque omnium sanctorum Dei, cum summa veneratione, consecraverunt... ». Il faut également mentionner l'existence d'un office de saint Marcellin dans un manuscrit du début du XII ${ }^{\mathrm{e}}$ siècle composé à l'abbaye de Redon et au Mont-Saint-Michel (Archivio Segreto Vaticano, ms lat. 9668, $\mathrm{f}^{\circ}$ 6-9). Sur ce manuscrit, voir AvRIL, François, "Notes sur quelques manuscrits bénédictins normands du XI ${ }^{\mathrm{e}}$ ou du $\mathrm{XII}^{\mathrm{e}}$ siècle ", Mélanges d'archéologie et d'histoire de l'École française de Rome, 76, 1964, p. 491-526. 
remarquera que dans ces deux domaines (la custodia et la fidelitas), le duc est présenté comme le successeur de Louis le Pieux ${ }^{91}$ : au XII ${ }^{\mathrm{e}}$ siècle, pour les moines de Redon, le modèle ducal ce n'est donc pas le prince ou le roi breton du haut Moyen Âge, mais bien l'empereur carolingien; la principauté bretonne est clairement incluse dans le royaume. On remarquera également que l'empereur est présenté comme soumis au pape, ce qui constitue une évidente révision grégorienne de la situation carolingienne ${ }^{92}$. On remarquera enfin qu'en mettant en scène, à travers la lettre apocryphe de Conan III, le renoncement du duc à sa custodia, les moines de Redon cherchent sans doute à légitimer l'appel à Rome et l'intervention du légat, mais dénoncent du même coup la faillite ducale, son incapacité à protéger l'abbaye et à s'inscrire dans les pas de ses illustres prédécesseurs.

En second lieu, la notice affirme que l'abbé a reçu du pape la liberté de choisir le prélat chargé de reconsacrer l'abbatiale et que ce choix se porta sur l'archevêque de Tours et non sur l'évêque de Vannes. La notice réaffirme ainsi la légitimité de la métropole tourangelle aux dépens de Dol, ce en quoi elle s'inscrit de nouveau résolument dans le cadre du royaume ${ }^{93}$. De manière certes seulement ponctuelle, puisqu'elle ne bénéficie pas de l'exemption, elle manifeste également son indépendance à l'égard de son évêque diocésain.

Au regard de tous ces éléments, il reste difficile d'établir ce qui s'est " réellement " passé. Plusieurs éléments me paraissent toutefois assez sûrs : 1-Sans être nécessairement officiellement pourvus du titre de custodes, les ducs exerçaient sur l'abbaye de Redon une sorte de protection et entretenaient avec elle des liens étroits, même si ceux-ci étaient loin d'être exclusifs dans un contexte de diversification des intercesseurs privilégiés des élites laïques. 2- En dépit de son immunité, l'abbaye et son patrimoine faisaient l'objet d'agressions de la part de seigneurs laïcs que le duc n'avait pas su réprimer. 3- Il y eut certainement à Redon, en 1127, une grande cérémonie de consécration présidée par le légat Gérard d'Angoulême et l'archevêque de Tours, qui apparut aux moines comme une occasion exceptionnelle de réaffirmer l'éminence de leur abbaye auprès des élites ecclésiastiques et laïques de la région. 4- L'abbaye s'imaginait alors en centre pro-pontifical et en capitale religieuse de la Bretagne. Par là, elle espérait sans doute intimider le pouvoir ducal et les évêques et conserver une puissance et un rayonnement que n'hésitaient pas à lui contester les autres institutions monastiques anciennes ou nouvelles.

91. Ibid. : « Reddo [Conan III] igitur vobis [Honorius II] eam liberam, sicut pius Ludovicus imperator liberam dedit..."

92. On a vu supra que l'auteur de la Vita sancti Conuuoinis procédait de même.

93. Remarquons que la notice se démarque alors de la position passée de certains papes (Grégoire VII, Pascal II par l'intermédiaire du légat Gérard d'Angoulême) qui avaient cru judicieux de contourner l'hostilité des archevêques de Tours à la réforme en promouvant le siège de Dol. On sait qu'à la fin du XII ${ }^{e}$ siècle, Innocent III mit définitivement un terme aux prétentions doloises. 
Cette espérance recelait-elle une part de vérité ou ne manifestait-elle qu'une ambition démesurée? Un événement postérieur d'une dizaine d'années permet d'avancer l'hypothèse qu'il ne s'agissait pas seulement d'une rêverie. Entre 1136 et 1140, une importante assemblée fut de nouveau réunie à Redon ${ }^{94}$. Elle était de nouveau présidée par l'archevêque de Tours et rassemblait les évêques de Quimper, Alet, Vannes et Nantes, ainsi que le duc Conan III et son entourage. Il semble qu'on y discuta essentiellement de la confirmation de la paix de Dieu sous l'égide du duc ${ }^{95}$. La Bretagne prenait ainsi place dans le cadre général de la réappropriation par les princes de la paix de Dieu, une réappropriation dont les ducs de Normandie et les comtes de Flandre avaient été les initiateurs et qui illustrait l'ascendant croissant exercé par les princes sur les élites ecclésiastiques de leur principauté. Il est par conséquent assez remarquable que ce soit à Redon et non dans une cité épiscopale, Rennes, Nantes ou Dol, que cette assemblée se tînt. Est-ce excessif de penser que ce choix s'explique par la reconnaissance de Redon comme le foyer privilégié d'une légitimité ducale fondée sur l'Église et même sur l'Église romaine?

94. Cette assemblée n'est connue que par une mention furtive dans un acte en faveur de l'abbaye de Saint-Méen : cf. Guillotel, Hubert, Actes des ducs de Bretagne, op. cit., p. 455.

95. L'assemblée fut réunie " pro pacis Dei confirmande intuitu ». Sur le renouveau de la paix de Dieu au XII ${ }^{\mathrm{e}}$ siècle, voir en dernier lieu CARRAZ, Damien, " Un revival de la paix de Dieu? Les paix diocésaines du XII ${ }^{\mathrm{e}}$ siècle dans le Midi ", dans La réforme " grégorienne "

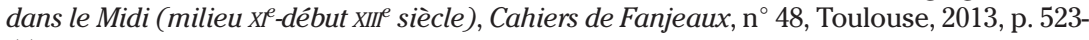
558 . 


\section{Excursus - La translation des reliques de saint Maixent et le devenir de l'abbaye de Redon au xe siècle}

La notice de peu postérieure aux 20-21 juin 924 relatant la translation des reliques de saint Maixent de Bretagne jusqu'à Poitiers, via la Touraine (Candé) et la Bourgogne (Auxerre), est la dernière source nous renseignant indirectement sur Redon avant les années 990 : à ce titre, elle constitue un témoignage irremplaçable sur la situation de la communauté monastique au $\mathrm{x}^{\mathrm{e}}$ siècle ${ }^{96}$. Cette notice ne nous est connue que par la copie qui en fut faite au début des années 1080 dans le cartulaire de Redon ${ }^{97}$. Elle a fait l'objet d'une excellente analyse par Guy de Poerck dans la Revue bénédictine dès $1962^{98}$. Malheureusement, cette analyse n'a pas connu le rayonnement qu'elle aurait mérité et fut curieusement négligée par Hubert Guillotel, dont le commentaire en partie erroné effectué dans son article sur l'exode du clergé breton devant les invasions scandinaves, publié dans les Mémoires de la Société d'histoire et d'archéologie de Bretagne en 1982, continue de faire autorité ${ }^{99}$. Il convient donc de reprendre les principaux éléments mis en lumière par Guy de Poerck en les complétant légèrement et d'en tirer toutes les conclusions au sujet du devenir de la communauté de Redon au X ${ }^{\mathrm{e}}$ siècle.

\section{Nature et analyse du texte}

Ce texte apparaît comme une notice composite, voire une notice-charte ${ }^{100}$. Il se présente en effet sous la forme d'un récit au passé et à la troisième personne de la translation des reliques de saint Maixent, en plusieurs étapes, de Bretagne jusqu'à Poitiers. Le récit commence avec l'arrivée en Bretagne d'un prêtre nommé Tutgual, envoyé par le vicomte Aimeric ${ }^{101}$ pour obtenir le retour à Poitiers du corps

96. $C R, \mathrm{n}^{\circ} 283$.

97. $\mathrm{F}^{\circ} 134 \mathrm{v}^{\circ}-135 \mathrm{v}^{\circ}$. La notice constitue l'un des derniers actes de la première phase rédactionnelle du cartulaire (laquelle s'arrête au fol. 139).

98. DE POERCK Guy, «Les reliques des saints Maixent et Léger aux IX et $\mathrm{X}^{\mathrm{e}}$ siècles et les origines de l'abbaye d'Ébreuil en Bourbonnais ", Revue bénédictine, 72/1-2, 1962, p. 61-95.

99. GuilLotel Hubert, "L'exode du clergé breton devant les invasions scandinaves ", Mémoires de la Société d'histoire et d'archéologie de Bretagne, 59, 1982, p. 269-300, en particulier p. 285-287 (l'auteur cite G. de Poerck dans sa note 95 mais ignore de fait son analyse).

100. Puisqu'après un long récit qui relève de la notice, celle-ci inclut quelques éléments d'une charte disparue. L'expression charte-notice utilisée par l'historiographie régionale est en revanche à proscrire.

101. Ce vicomte appartient à l'entourage du comte Èbles de Poitiers (PRELL, Jan Hendrik, L'entourage des comtes du Poitou, ducs d'Aquitaine, de 902 à 1030, mémoire de maîtrise dactyl., Poitiers, 1984). Il est vraisemblablement le frère cadet de Savaric, vicomte [de Thouars] : cf. JEANNEAU, Cédric, " Émergence et affirmation des familles seigneuriales à la frontière des principautés territoriales. Les seigneurs de la Garnache et les vicomtes de Thouars ", dans AURELL, Marc (dir.), Le médiéviste et la monographie familiale : sources, méthodes et problématiques, Turnhout, 2004, p. 161-187, p. 183 et COLLET, Jean-Philippe, Les vicomtes de Thouars (IXe siècle-1154), l'ascension d'un lignage au temps des principautés, entre comtes du Poitou et comtes d'Anjou, mémoire de maîtrise dactyl., Université de Poitiers, 1998; même si certains situent plutôt son pouvoir du côté de Loudun : BEECH, George, " the Origins of the family of the viscounts of Thouars ", dans Études de Civilisation médiévale (IXe-XII siècles). Mélanges offerts à E.-R. Labande, Poitiers, 1974, p. 25-31, suivi par Damon, Géraldine, "Vicomtes et vicomtés dans le Poitou médiéval (IXe-XII siècle). 
de saint Maixent. Plélan, où nous savons que les reliques de Maixent étaient conservées depuis le milieu des années $860^{102}$, n'est pas nommé, ni Redon dont Plélan dépendait. Après une négociation menée avec le doyen Moroc, dont nous pouvons supposer qu'il était responsable de la cella de Plélan, Tutgual repart pour le Poitou avec les reliques, accompagné par un petit groupe de moines bretons auxquels leur garde a été confiée (au moins pour la durée du voyage). La petite troupe se dirige d'abord vers la Loire, puis, alertée par la nouvelle que des " païens " dévastaient le Poitou ${ }^{103}$, elle repart vers l'est pour s'établir à Candé, où elle fait l'acquisition d'une église et réside un certain temps ${ }^{104}$. La menace des " Normands " se rapprochant, le groupe finit par repartir pour le pagus d'Auxerre, répondant à l'appel du duc Richard (le Justicier), alors encore en vie - ce qui place cet événement entre 911 et le 31 août ou le $1^{\text {er }}$ septembre $921^{105}$ - qui lui offrait sa protection ${ }^{106}$. Là, l'évêque et les grands de la région lui proposèrent de nombreux "bénéfices, fiscs et domaines " pour l'inciter à s'établir définitivement ${ }^{107}$. Mais désireux d'honorer leur promesse initiale, les moines dépêchèrent deux représentants à Poitiers ${ }^{108}$, avec le prêtre Tutgual, pour négocier avec le vicomte Aimeric. Devant le comte Ėbles ${ }^{109}$, un arbitrage fut alors conclu, sous l'égide de l'archidiacre et du doyen du chapitre cathédral de Poitiers, entre d'une part le vicomte Aimeric, avoué de l'abbaye Saint-Maixent ${ }^{110}$, et son frère Adhémar qui en était l'abbé ${ }^{111}$, d'autre part les moines venus de Bretagne. Les moines s'engagèrent à rapporter à Poitiers le corps de saint Maixent, ainsi que sa tête à l'exception de la mâchoire maxillaire, avec sa Vita et son missel. En contrepartie, ils obtenaient la garde des reliques à perpétuité et sous la protection du comte ${ }^{112}$, et recevaient d'Aimeric et de l'abbé Adhémar des vivres (probablement pour tenir jusqu'à la prochaine récolte) et un moulin, et de la part d'Aimeric seulement " une terre culte et inculte " à partager équitablement. Les moines pouvaient,

Genèse, modalités et transformations ", dans Vicomtes et vicomtés dans l'Occident médiéval, Toulouse, 2008, p. 223-235.

102. $C R, \mathrm{n}^{\circ} 241$.

103. $C R, \mathrm{n}^{\circ} 283$ : " [...] audivimus quod pagani devastabant Pictavensem regionem..."

104. Candé-sur-Beuvron (ch.-l. c., Loir-et-Cher), en Blésois.

105. Avant 911, la région d'Auxerre était encore ravagée par les Normands et le duc Richard est mort le 31 août 921 (cf. Les Annales de Flodoard, éd. Philippe LAUER, Paris, 1906, p. 5 et la Chronique de Saint-Pierre-le-Vif, éd. Robert-Henri BauTiER et Monique GiLles, Paris, 1979, p. 70). Sur l'autorité de Richard en Auxerrois, voir SASSIER Yves, Recherches sur le pouvoir comtal en Auxerrois du Xe au début du XIII siècle, Paris, 1980, p. 3-15.

106. $C R, \mathrm{n}^{\circ} 283$ : «Devastatio autem Normannorum et timor crevit super nos quatinus ad salvamentum diffinivimus deportare corpus ipsius viri, et ita fecimus, in Autisiodorum pagum deportantes honorifice cum adjutorio Ricardi comitis tunc temporis in corpore viventis. "

107. Ibidem : "Ille vero cum episcopo et optimatibus totius regionis plurima beato viro beneficia et fiscos et predia libenti animo ac devote donare voluerunt."

108. Ibid. : " [...] et ideo Mesetum monachum ac prepositum et Joseph monachum ac sacerdotem ad eum misimus Pictavim... " Ce qui permet de constater que le doyen Moroc était resté à Plélan et que le petit groupe parti avec Tutgual s'était doté, avec Meset, de son propre doyen.

109. Ėbles Manzer, comte de Poitiers (902-934).

110. Aujourd'hui Saint-Maixent-l'École (ch-l. c., Deux-Sèvres), à une cinquantaine de km au sud-ouest de Poitiers.

111. Sur Adhémar, abbé de Saint-Maixent dès 903 et jusqu'en 928 au moins, voir la notice de RICHARD Alfred, Chartes et documents pour servir à l'histoire de l'abbaye de Saint-Maixent, Poitiers, 1886, p. LXIV-LXV et DE POERCK Guy, "Les reliques des saints Maixent... ", art. cit., p. $64-65$, note 4 .

112. $C R, \mathrm{n}^{\circ} 283:$ : [...] et nos simus ipsius corporis sine fine custodes, cum auctoritate Eubuli comitis et securitate..." 
s'ils le souhaitaient, revêtir l'habit des moines de Saint-Maixent et se placer sous l'autorité d'Adhémar. Ces dispositions furent confirmées sous serment le dimanche 20 juin 924 dans l'église Sainte-Marie in canonica beati Petri (l'enclos canonial de la cathédrale de Poitiers), puis de nouveau le lendemain en présence du comte. La notice reprend alors quelques éléments de l'eschatocole de la charte qui fut alors rédigée - une liste de signa et la formule de datation - puis elle s'achève, de manière lacunaire, par un éloge du comte, "tutor [...], defensor ac patronus".

\section{L'auteur}

L'auteur de la notice demeure anonyme, mais il s'agit à l'évidence d'un moine breton appartenant à la communauté de Plélan ${ }^{113}$, qui accompagne les reliques de saint Maixent tout au long de leur périple en compagnie de quelques autres moines et du prêtre Tutgual, dépêché par le vicomte Aimeric. Au terme de ces pérégrinations, lui et ses compagnons sont confirmés dans la garde des reliques et placés sous l'autorité de l'abbé de Saint-Maixent Adhémar, ce qui explique pourquoi celui-ci est parfois désigné par l'auteur, de manière rétrospective, comme "notre abbé ${ }^{114}$ ". C'est seulement une fois installé à Poitiers avec ses compagnons, donc après les 20-21 juin 924, que l'auteur entreprit de raconter cette histoire qui s'étale donc sur plusieurs années.

\section{Interprétation et enjeux historiques}

À l'origine de la translation se trouve clairement la volonté d'un groupe familial de l'aristocratie poitevine engagé dans la restauration de l'abbaye Saint-Maixent et désireux, pour en rehausser le prestige et le rayonnement, de récupérer les reliques du saint éponyme de leur établissement qui se trouvaient en Bretagne. À la tête de ce groupe figurent le vicomte Aimeric et son frère Adhémar, respectivement avoué et abbé de Saint-Maixent ${ }^{115}$. Ils bénéficient du soutien du comte de Poitiers Èbles, qui mobilise également à leur profit le chapitre cathédral de sa cité, et ont peut-être aussi joui de la bienveillance du comte de Rennes Bérenger, dont on sait qu'il entretînt de bonnes relations avec le fils d'Èbles dans les années 940. Dans ce cadre, le vicomte Aimeric envoya en Bretagne une ambassade en la personne du prêtre Tutgual, dont le nom indique une origine bretonne sans doute perçue comme propice à la réussite des négociations à mener avec les moines locaux. De ces derniers nous ne savons presque rien : nous avons vu que ni Plélan, ni Redon ne sont nommés; la notice nous apprend que Tutgual négocia avec le doyen Moroc, sans doute responsable de la dépendance de Plélan sous l'autorité de l'abbé de Redon et qui est probablement le même homme que ce moine Moroc apparaissant dans un acte de Redon daté de $913^{116}$. Ce doyen semble disposer d'une assez grande marge de manœuvre puisqu'il négocie le sort des reliques de sa dépendance sans en référer à l'abbé et qu'après avoir donné son accord à leur départ en échange de la promesse de recevoir de " nombreux cadeaux ou bénéfices " de la part du vicomte

113. Au début du récit, l'auteur désigne le doyen Moroc, avec lequel Tutgual mène les négociations, comme son doyen. : " [...] adiit Tutgualos sacerdos... causa corporis beati Maixentii ad nos et locutus est cum nostro decano nomine Moroc..."

114. $C R, \mathrm{n}^{\circ} 283$ : "Haemericus autem et noster abbas promiserunt nobis..."

115. Leur action se poursuit les années suivantes : cf. RICHARD Alfred, Chartes et documents... de Saint-Maixent, op. cit., ${ }^{\circ} 11$ (925).

116. $C R, \mathrm{n}^{\circ} 277$ (datation corrigée par Hubert Guillotel). 
Aimeric, il décide, de nouveau seul apparemment, d'envoyer plusieurs moines pour accompagner les reliques jusqu'en Poitou. Ces derniers finiront par se placer sous l'autorité de l'abbé de Saint-Maixent tout en bénéficiant, au moins temporairement, d'une autonomie relative facilitée par leur installation à Poitiers même - on sait grâce à une source locale que les reliques de saint Maixent ne réintégrèrent l'abbaye Saint-Maixent que dans les années suivantes, avant $942^{117}$. La translation de reliques s'accompagna donc du transfert d'un petit groupe de moines, issus de la dépendance de Plélan, de la communauté de Redon à la communauté de Saint-Maixent. L'abbé de Saint-Maixent tenta même, semble-t-il, de s'approprier la dépendance de Plélan - c'est le sens de la possibilité laissée aux moines restés en Bretagne de revêtir l'habit de Saint-Maixent ${ }^{118}$-, sans succès cependant puisque Plélan demeura dans le patrimoine de Redon et en devint par la suite un prieuré ${ }^{119}$.

Une telle compréhension des événements appelle plusieurs remarques. En premier lieu, il est clair que le départ des reliques de Plélan n'est pas lié à la menace que les Normands auraient fait peser sur Redon, Plélan ou la Bretagne en général. Les ravages des " païens " et la " devastatio Normannorum et timor " ne sont évoqués qu'après le départ de Bretagne, d'abord lorsque la petite troupe doit renoncer à gagner directement le Poitou qui, lui, est alors parcouru par les Normands - certainement les Normands de la Loire établis dans la région de Nantes depuis les années 860 -, ensuite lorsque la pression exercée par ces derniers la pousse à fuir son refuge temporaire de Candé sur la Loire pour aller plus loin vers l'est. Les Normands ne sont donc pas la cause de la translation des reliques de saint Maixent, même s'ils en expliquent pour partie les pérégrinations. On ne peut par ailleurs rien tirer de la notice au sujet de leur action en Bretagne même, pas plus que l'on ne peut dater le départ des moines de Plélan de 919 ou 920 sur la seule base de mentions annalistiques évoquant de manière générale des raids normands en Bretagne à cette date ${ }^{120}$.

En revanche, la translation du corps de saint Maixent s'inscrit parfaitement dans le cadre de la " politique des reliques " que mènent les grands de l'aristocratie post-carolingienne pour doter les établissements de leurs domaines ou principautés des trésors de reliques aptes à assurer la protection et à apporter la prospérité aux populations placées sous leur autorité ${ }^{121}$. Cette politique des reliques explique d'ailleurs aussi certains aléas des translations, comme on le voit ici avec l'intervention du duc Richard qui tente de contrecarrer les efforts du vicomte Aimeric et du comte Èbles et d'attirer les reliques de saint Maixent en Bourgogne avec le soutien des élites ecclésiastiques et laïques du pagus d'Auxerre. La destinée des reliques de

117. La notice ne précise pas où dans Poitiers, ni dans quelle église furent abritées les reliques de saint Maixent confiées à la garde des moines venus de Bretagne. Mais leur présence n'est pour la première fois attestée dans l'abbatiale de Saint-Maixent qu'avec une charte de donation de février 942 : cf. RichaRD, Alfred, Chartes et documents... de Saint-Maixent, op. cit., $\mathrm{n}^{\circ} 16:$ : [...] ad basilicam sancti Maxentii ubi ipse corpore quiescit." 118. $C R, \mathrm{n}^{\circ} 283$ : " [...] et si quis ex nostratibus talem habitum indui voluerit, nullatenus sit preocupatus si utilitas tamen ejus a pluribus sit vel fuerit comprobata..."

119. Bulle d'Eugène (1147), éd. Papsturkunden in Frankreich..., op. cit., n 253, : " obedientia ecclesie Sancti Salvatoris de Plebelan ".

120. Contra Guillotel Hubert, "L'exode du clergé breton... ", art. cit., p. 285-286, d'après les Annales de Flodoard (919). Un passage des Annales dites de Redon évoque également des attaques normandes en " petite Bretagne " en 920 (bibl. d'Avranches, ms 213, $\mathrm{f}^{\circ} 172$, éd. Philippe LABBÉ, Nova bibliotheca manuscriptorum librum..., Paris, 1657, t. I, p. 349-350 : notons qu'il s'agit d'un manuscrit du Xve siècle du Mont-Saint-Michel, que rien ne rattache explicitement à Redon).

121. Bozoky Edina, La politique des reliques de Constantin à Saint Louis, Paris, 2006. 
saint Maixent n'est à ce titre guère différente de celle des reliques de saint Philibert, qui errèrent également d'une protection aristocratique à l'autre dans les décennies 830-870, de l'île de Noirmoutier à l'Herbauge, de l'Herbauge à l'Anjou, de l'Anjou au Poitou, du Poitou à l'Auvergne, de l'Auvergne à la Bourgogne ${ }^{122}$.

Les éléments de la négociation aboutissant à la cession des reliques de saint Maixent au vicomte Aimeric nous échappent toutefois complètement. La contrepartie évoquée par la notice, à savoir les nombreux beneficia promis par le vicomte au doyen Moroc, ne convainc pas vraiment ${ }^{123}$. S'il est fait référence ici à des objets matériels, leur évocation serait étonnante car elle ferait peser sur la cession des reliques un parfum de simonie ${ }^{124}$. Si l'expression désigne des terres données en bénéfice, on ne voit pas bien comment un vicomte de Poitiers aurait pu en concéder en Bretagne (seule région où cela aurait pu intéresser les moines de Plélan) où il n'avait ni droit ni terre; il faudrait donc y voir une promesse de concession en Poitou et donc imaginer que le doyen Moroc envisageait la fondation d'une dépendance très éloignée de la Bretagne dans un contexte où, comme le montrent les pérégrinations des reliques de saint Maixent elles-mêmes, les relations au sein des régions atlantiques n'étaient pas faciles ${ }^{125}$. Quoi qu'il en soit, la promesse de beneficia ne suffit pas à expliquer un abandon aussi préjudiciable à la communauté de Plélan. On est donc conduit à faire l'hypothèse de motifs sous-jacents plus contraignants. À ce propos, il faut souligner d'une part que les reliques de saint Maixent n'étaient présentes à Plélan que depuis somme toute assez peu de temps (40 à 50 ans), d'autre part que les conditions de leur arrivée restent obscures et qu'il est fort possible que le roi Salomon, qui les utilisa dans son projet de fondation d'une nouvelle communauté monastique dans son palais de Plélan dans les années 860 , les ait préalablement acquises de manière plus ou moins opportuniste, en proposant aux moines de Saint-Maixent, alors en fuite devant les Normands, de les accueillir sur ses terres ${ }^{126}$ ou même en leur dérobant leurs reliques à l'occasion d'un furtum sacrum dont on sait qu'ils étaient alors fréquents ${ }^{127}$. Au tournant des années 910-920, dans un nouveau contexte géopolitique marqué par l'affaiblissement de la Bretagne, les grands poitevins auraient pu obtenir du comte de Rennes, dans le cadre de négociations plus larges, qu'il convainque les moines de Redon d'accepter le retour des reliques de saint Maixent dans leur région d'origine, et ces derniers auraient pu d'autant plus facilement y consentir que cette cession concernait surtout Plélan, une simple dépendance qui avait tenté jadis d'acquérir une certaine autonomie. Tout cela n'est qu'hypothèse, mais il est intéressant de noter à ce propos qu'au XI ${ }^{\mathrm{e}}$ siècle l'auteur de la Vita Conwoionis tenta de trouver une meilleure et bien plus classique justification

122. CARTRON Isabelle, Les pérégrinations de Saint-Philibert. Genèse d'un réseau monastique dans la société carolingienne, Rennes, 2009.

123. $C R, \mathrm{n}^{\circ} 283$ : "[...] promittens nobis plurima beneficia ex parte Haemerici et nos ei credidimus..."

124. La question de la simonie a agité les affaires bretonnes dans la seconde moitié du IX ${ }^{\mathrm{e}}$ siècle : cf. ChÉDEVILLE André et GuILlOTEL Hubert, La Bretagne des saints et des rois, $V^{e}-X^{e}$ siècle, Rennes, 1984, p. 266-273, 299-311.

125. L'époque est plutôt à la rétractation des anciens grands domaines monastiques de l'époque carolingienne.

126. Les reliques de saint Maixent étaient encore à l'abbaye Saint-Maixent en 848. Entre 853 et 865/866, en raison de la menace normande, elles furent emportées par les moines poitevins jusqu'à Ébreuil, en Bourbonnais. Avant le 17 avril 869, elles étaient passées entre les mains du roi Salomon qui les avait offert à sa nouvelle fondation de Plélan $\left(C R, \mathrm{n}^{\circ} 241\right)$. Cf. DE Poerck Guy, "Les reliques des saints Maixent... ", art. cit., p. 91-95.

127. Geary Patrick, Le vol des reliques au Moyen Âge, Paris, 1993. 
à la présence des reliques de saint Maixent à Plélan que le don de Salomon, ce qui reflète un certain malaise vis-à-vis de l'action de ce souverain : selon lui, le saint serait tout simplement venu finir ses jours à Plélan à l'issue de sa longue carrière ${ }^{128}$.

Une telle relecture du dossier permet de nuancer les certitudes communément admises au sujet de la situation de Redon au $\mathrm{x}^{\mathrm{e}}$ siècle. La notice ne concerne pas la communauté monastique installée à Redon, mais seulement les moines de la dépendance de Plélan. On ne saurait donc fonder sur elle la thèse d'un exil des moines de Redon hors de Bretagne ${ }^{129}$. En outre, seuls quelques moines de Plélan, parmi lesquels l'auteur de la notice, le doyen Meset, le prêtre Joseph, le moine Gleuhoiarn, participèrent au voyage et finirent par s'établir à Poitiers et par changer d'obédience ${ }^{130}$. Le site de Plélan ne fut pas abandonné et resta apparemment sous la gouverne du doyen Moroc et dans la dépendance de Redon. En revanche, le prestige jadis acquis grâce à la politique de Salomon n'était plus qu'un lointain souvenir. L'absence d'actes concernant Redon entre les années 910/913 et la fin du $\mathrm{x}^{\mathrm{e}}$ siècle n'en reste pas moins intrigante, mais on ne peut la mettre sur le compte d'un abandon du site ou d'une déliquescence de la communauté liés aux raids normands des premières décennies du siècle. D'autant que la communauté de Redon fut capable, comme on l'a vu, de conserver ses archives et son patrimoine constitués au $\mathrm{IX}^{\mathrm{e}}$ siècle.

Il reste à expliquer la copie dans le cartulaire de Redon d'un texte attestant la perte de précieuses reliques, car en général on ne se vante pas de pareille mésaventure $^{131}$. L'hypothèse la plus probable se place dans la continuité des remarques précédentes. Ce récit, qui permettait déjà d'expliquer la perte des reliques données jadis par Salomon - le cartulaire contient la donation de $869^{132}-$, n'affectait pas l'abbaye de Redon, mais facilitait au contraire le renforcement de son autorité sur un établissement qui avait pu un temps la concurrencer. Ayant perdu le corps du saint et ayant vu une partie de ses membres absorbés par le monastère Saint-Maixent de Poitiers, Plélan n'était plus qu'un prieuré de Redon parmi d'autres.

128. Vita sancti Conwoionis, éd. BRETT Caroline, The Monks of Redon, op. cit., p. 245 : « [...] iuxta beatum ex Pictauensi territorio abbatem Maxentium, qui olim molestias falsorum sui monasterii fratrum fugiens illuc uenerat, ibique ad Dominum migrauerat. "

129. Contra GuilLotel Hubert, "L'exode du clergé breton... ", art. cit.

130. CR, $\mathrm{n}^{\circ} 283$ : "[...] sig. Mesetus prepositus, sig. Joseph monachus [...] sig. Gleuhoiarnus..."

131. Il est probable que l'original de la notice ou une autre copie se trouvait à SaintMaixent de Poitiers, mais les archives de cette abbaye ont énormément souffert des outrages du temps : cf. RichARD Alfred, Chartes et documents... de Saint-Maixent, op. cit., p. XIII-XXII.

132. $C R, \mathrm{n}^{\circ} 241$. 


\section{RÉSUMÉ}

À partir d'une analyse croisée de la Vita Conwoinis, du cartulaire de Redon et des épaves du " petit cartulaire ", cette étude envisage la manière dont la communauté de Redon des $\mathrm{XI}^{\mathrm{e}}$-XII ${ }^{\mathrm{e}}$ siècles concevait son passé et entendait se situer dans son environnement politique, social et institutionnel. Il apparaît que les stratégies discursives mises en œuvre par les moines reposent sur une mise en valeur orientée de l'héritage carolingien et la célébration de l'engagement de leur abbaye dans la réforme de l'Église promue conjointement par la papauté et un corps épiscopal rénové. Ces choix conduisent les moines de l'âge grégorien à minimiser le rôle des ducs de Bretagne, qui

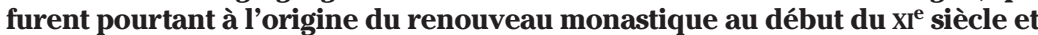
firent de l'abbaye un haut lieu de leur pouvoir tout au long des $\mathrm{XI}^{\mathrm{e}}$-XII ${ }^{\mathrm{e}}$ siècles.

\section{ABSTRACT}

Through cross analysis of the Vita Conwoinis, of the Redon cartulary and of the remains of the "small cartulary", this study seeks to determine the manner in which the Redon community of the eleventh and twelfth centuries perceived its past and sought to place itself in the wider political, social and institutional context. It seems that the discursive strategy employed by the monks was based on valorising a biased conception of the Carolingian legacy and celebrating the commitment of their abbey to Church reform as promoted by the papacy and the renewed episcopal corps. These choices led the monks of the Gregorian age to minimise the role of the dukes of Britany, despite the fact that they had initiated monastic renewal at the beginning of the eleventh century and had made the abbey into one of the major centres of their power throughout the eleventh and twelfth centuries. 